\title{
Protein tyrosine phosphatase nonreceptor type 2 controls colorectal cancer development
}

\author{
Egle Katkeviciute, ${ }^{1}$ Larissa Hering, ${ }^{1}$ Ana Montalban-Arques, ${ }^{1}$ Philipp Busenhart, ${ }^{1}$ Marlene Schwarzfischer, ${ }^{1}$ Roberto Manzini, ${ }^{1}$ \\ Javier Conde, ${ }^{1}$ Kirstin Atrott, ${ }^{1}$ Silvia Lang, ${ }^{1}$ Gerhard Rogler, ${ }^{1,2}$ Elisabeth Naschberger, ${ }^{3}$ Vera S. Schellerer, ${ }^{4}$ Michael Stürzl, ${ }^{3}$ \\ Andreas Rickenbacher, ${ }^{5}$ Matthias Turina, ${ }^{5}$ Achim Weber, ${ }^{6}$ Sebastian Leibl, ${ }^{6}$ Gabriel E. Leventhal, ${ }^{7}$ Mitchell Levesque, ${ }^{8}$ \\ Onur Boyman, ${ }^{9,10}$ Michael Scharl, ${ }^{1,2}$ and Marianne R. Spalinger ${ }^{1}$ \\ DDepartment of Gastroenterology and Hepatology, University Hospital Zurich, and '2Zurich Center for Integrative Human Physiology, University of Zurich, Zurich, Switzerland. ${ }^{3}$ Division of Molecular and \\ Experimental Surgery, and ${ }^{4}$ Department of Surgery, University Medical Center of Erlangen, Friedrich-Alexander-University Erlangen-Nürnberg, Erlangen, Germany. ${ }^{5}$ Department of Visceral and Transplant \\ Surgery, and ${ }^{6}$ Department of Pathology, University Hospital Zurich, Zurich, Switzerland. Department of Civil and Environmental Engineering, Massachusetts Institute of Technology (MIT), Cambridge, \\ Massachusetts, USA. ${ }^{8}$ Department of Dermatology and ${ }^{9}$ Department of Immunology, University Hospital Zurich, Zurich, Switzerland. ${ }^{10}$ Faculty of Medicine, University of Zurich, Zurich, Switzerland.
}

Protein tyrosine phosphatase nonreceptor type 2 (PTPN2) recently emerged as a promising cancer immunotherapy target. We set out to investigate the functional role of PTPN2 in the pathogenesis of human colorectal carcinoma (CRC), as its role in immune-silent solid tumors is poorly understood. We demonstrate that in human CRC, increased PTPN2 expression and activity correlated with disease progression and decreased immune responses in tumor tissues. In particular, stage II and III tumors displayed enhanced PTPN2 protein expression in tumor-infiltrating T cells, and increased PTPN2 levels negatively correlated with expression of PD-1, CTLA4, STAT1, and granzyme A. In vivo, T cell- and DC-specific PTPN2 deletion reduced tumor burden in several CRC models by promoting CD44+ effector/memory T cells, as well as CD8 ${ }^{+} \mathrm{T}$ cell infiltration and cytotoxicity in the tumor. In direct relevance to CRC treatment, T cell-specific PTPN2 deletion potentiated anti-PD-1 efficacy and induced antitumor memory formation upon tumor rechallenge in vivo. Our data suggest a role for PTPN2 in suppressing antitumor immunity and promoting tumor development in patients with CRC. Our in vivo results identify PTPN2 as a key player in controlling the immunogenicity of CRC, with the strong potential to be exploited for cancer immunotherapy.

\section{Introduction}

Colorectal carcinoma (CRC) is the third most frequent malignancy and the second leading cause of cancer-related deaths worldwide $(1,2)$. In 2018 , more than 1.8 million patients were newly diagnosed with CRC, and despite improved 5-year survival rates, the estimated number of CRC-related deaths accumulated to 881,000 (3-6). Successful immunotherapeutic interventions in other solid cancers, such as melanoma and lung carcinoma, have exploited the tumor microenvironment, which contains high numbers of immune cells, such as T cells, NK cells, and antigen-presenting cells $(7,8)$. The majority of CRCs, however, lack prominent immune infiltrates and only approximately $4 \%$ of all patients with CRC respond to immunotherapies $(2,9)$. Immunogenic CRCs are characterized by mismatch repair deficiency and high microsatellite instability resulting in a high tumor mutation rate (2). In contrast, the majority of CRC tumors exhibit low immunogenicity and evade eradication by circulating T cells and NK cells via expression of immunosuppressive modulators such as TGF- $\beta$, recruitment of Tregs, and promotion of myeloid-derived suppressor cells (10). This absence of antitumor

Conflict of interest: The authors have declared that no conflict of interest exists. Copyright: (5) 2021, American Society for Clinical Investigation.

Submitted: May 14, 2020; Accepted: September 29, 2020; Published: January 4, 2021.

Reference information: / Clin Invest. 2021;131(1):e140281.

https://doi.org/10.1172/JCl140281. immune cell recruitment is the main hurdle for successful immunotherapy and generates a high need for effective immune modulators to render colorectal tumors more immunogenic (2).

Protein tyrosine phosphatase nonreceptor type 2 (PTPN2) has recently been suggested as a possible target for tumor therapy (11-13). PTPN2 is a ubiquitously expressed antiinflammatory protein known to regulate proinflammatory signaling pathways by direct dephosphorylation of several JAKs, STAT family members, and others (14-17). Total body deficiency of PTPN2 in mice causes severe systemic inflammation and death within 3-5 weeks of birth. Therefore, mice with cell type-specific PTPN2 deletions have been developed (18).

We have recently shown that loss of PTPN2 in myeloid cells and intestinal epithelial cells reduce tumor load and promote immune responses against tumors in a model of colitis-associated colon cancer by increasing IL- $1 \beta$ production and elevating immunogenicity of the tumor (19). Tumor cell-specific and $\mathrm{T}$ cell-specific PTPN2 deletion has been reported to reduce tumor load in s.c. tumor models using melanoma and CRC cell lines as well as in mammary tumor models (11-13). In humans, reduced PTPN2 expression correlated with a better treatment response in $\mathrm{T}$ cell acute lymphoblastic leukemia in children (20). However, the molecular role of PTPN2 in T cells in the context of difficult-to-treat solid tumors has not been studied in depth, and little is known about its role in human CRC. 
A
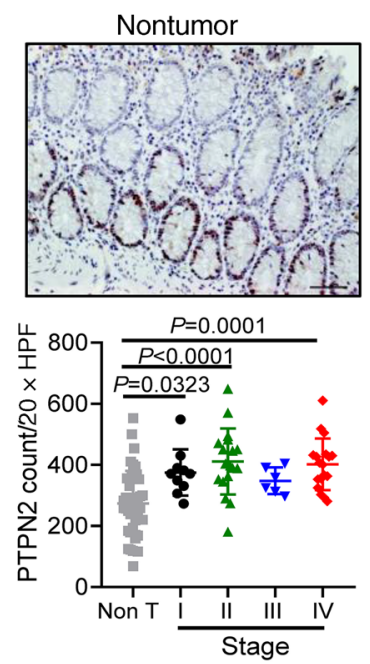

D

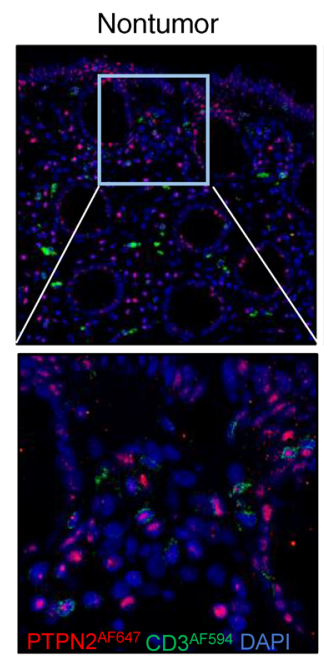

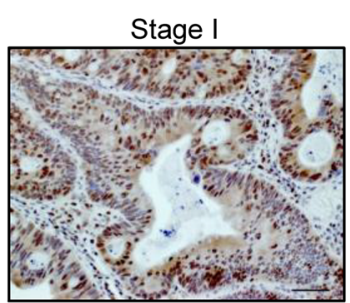

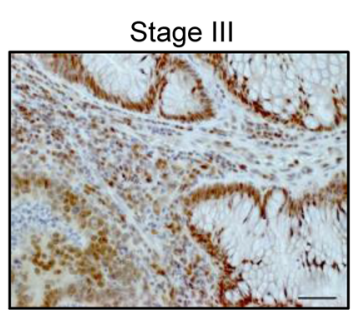

Stage I
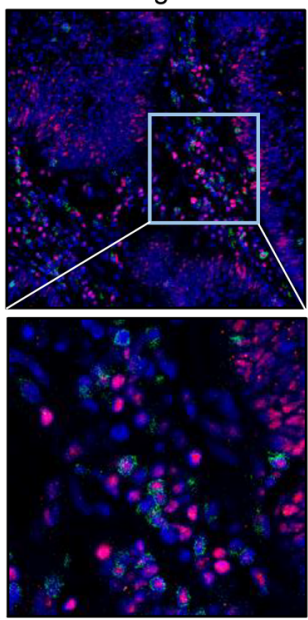

B

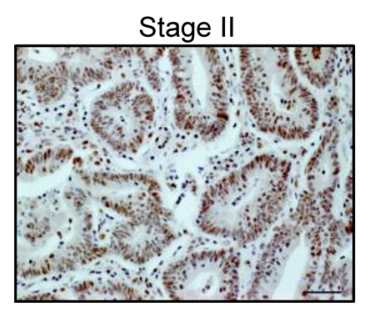

Stage IV

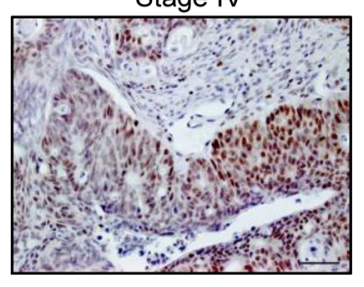

C

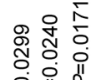

iil in
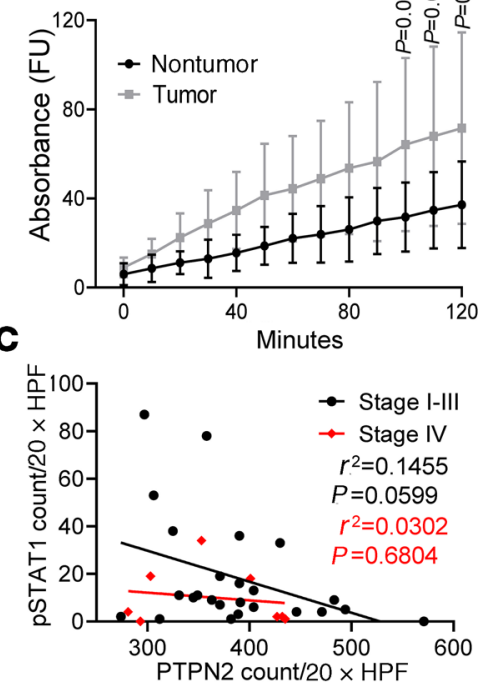

Stage II
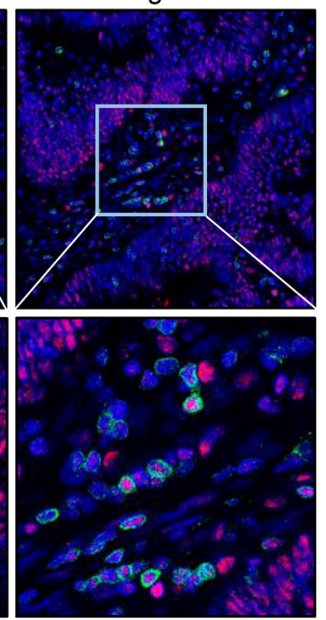

Stage III
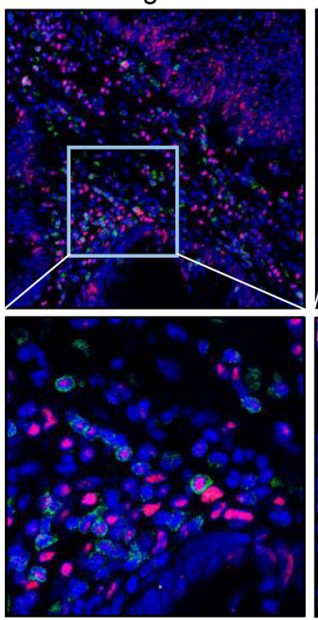

Stage IV

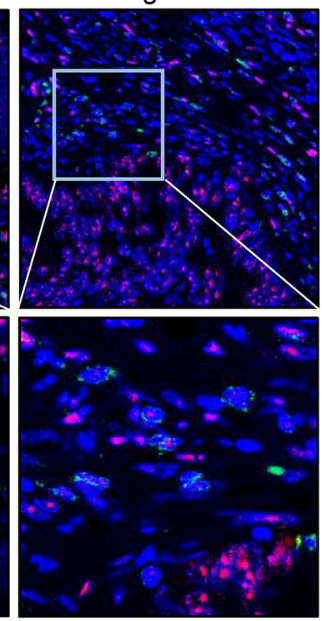

E
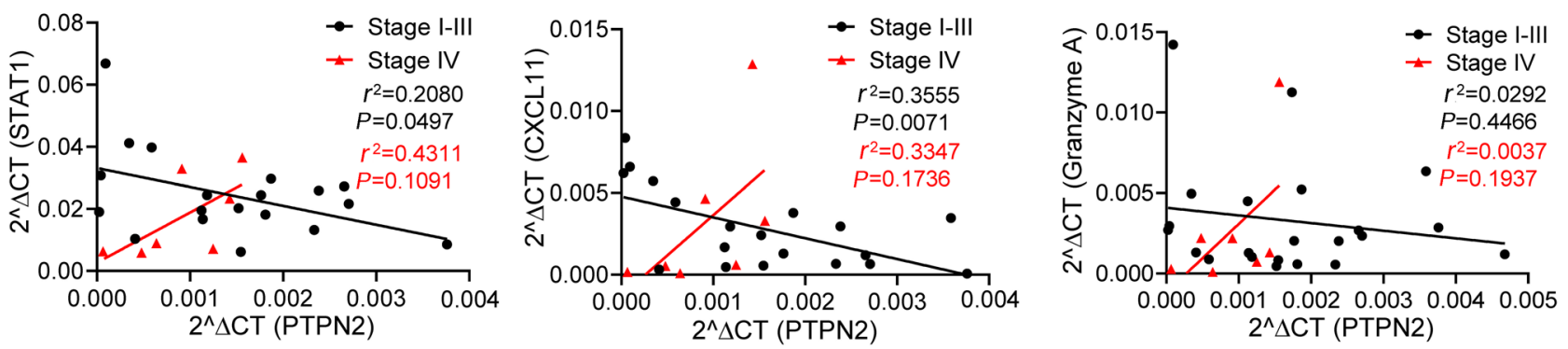

$\mathbf{F}$
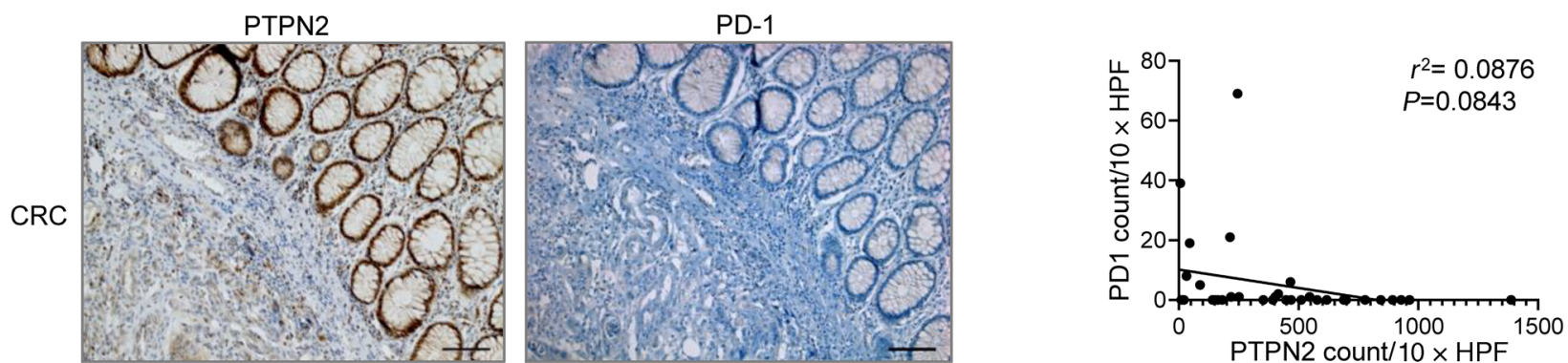
Figure 1. Enhanced PTPN2 expression in human CRC. (A) Representative images and quantification of PTPN2 IHC staining in nontumor (Non T) and stage I-IV tumor tissue. Scale bars: $50 \mu \mathrm{m}$. $P$ values were determined by 1-way ANOVA with Tukey's multiple-comparison test. Data represent the mean \pm SD. (B) PTPN2 phosphatase activity in nontumor and tumor tissue ( $n=10$ samples per condition). $P$ values were determined by 2 -tailed Mann-Whitney $U$ test. (C) Correlation between PTPN2 and p-STAT1 protein quantification and (D) immunofluorescence costaining for CD3 (AF594, green), PTPN2 (AF647, red), and DAPI. Original magnification, 40x; zoom factor, 3.0. $P$ and $R^{2}$ values in C were determined by linear regression analysis. (E) Correlations between PTPN2 and STAT1 and between CXCL11 and GZMA mRNA expression. $P$ and $R^{2}$ values were determined by linear regression analysis. (F) Representative images of $\mathrm{IHC}$ and correlations between PTPN2 and the checkpoint molecule PD-1 in primary CRC. Scale bars: $100 \mu \mathrm{m}$. $P$ values and $R^{2}$ values were determined by linear regression analysis. HPF, high-power field.

Here, we demonstrate that increased PTPN2 expression in human CRC tissue, and especially in infiltrating lymphocytes, correlates with reduced levels of $\mathrm{T}$ cell activity and cytotoxicity. Further, we show that PTPN2 deletion in T cells lowers tumor load in orthotopic and heterotopic CRC and melanoma mouse models and enhances $\mathrm{T}$ cell activity, infiltration, and cytotoxicity, thus rendering tumors more immunogenic. Finally, PTPN2 deletion in $\mathrm{T}$ cells potentiates the efficacy of anti-PD-1 treatment, resulting in more effective tumor clearance and immune memory formation. These data indicate that PTPN2 may be a very promising standalone or combination therapy target in CRC.

\section{Results}

PTPN2 expression is increased in patients with CRC. To investigate the importance of PTPN2 in the pathogenesis of human CRC, we first analyzed PTPN2 protein levels in colonic nontumor versus tumor tissues from patients with CRC using IHC. Although PTPN2 expression in nontumor tissues was moderate, we observed significantly enhanced levels of PTPN2 in CRC tumor tissues at all stages (Figure 1A). We found that PTPN2 phosphatase activity was increased in CRC tumor tissues compared with nontumor tissues from the same patient (Figure 1B). Consistent with enhanced PTPN2 activity, the levels of phosphorylated STAT1 (p-STAT1), a direct PTPN2 target, were low and inversely correlated with PTPN2 protein expression (Figure 1C).

PTPN2 was mainly expressed in tumor cells, but interestingly, in stages I-III, we observed increasing numbers of $\mathrm{CD}^{+}$cells, which were positive for PTPN2, whereas PTPN2 expression levels in the cancer cells did not change across the tumor stages. Stage IV tissue, however, showed lower numbers of PTPN2 ${ }^{+}$infiltrating $\mathrm{CD}^{+}$cells (Figure 1D), probably because of the overall reduced levels of $\mathrm{CD}^{+}$cells. Since PTPN2 and p-STAT1 are suggested to modulate $\mathrm{T}$ cell recruitment and activity $(11,21)$, we investigated whether PTPN2 expression correlates with $\mathrm{T}$ cell-associated genes, such as STAT1, CXCL11, and granzyme A (GZMA), which are required for $\mathrm{T}$ cell activity, recruitment, and cytotoxicity, respectively. We observed an inverse correlation in stages I-III for the ratios of STAT1/PTPN2, CXCL11/PTPN2, and GZMA/PTPN2 (Figure 1E). Stage IV tumors, however, showed a direct correlation for all 3 genes (Figure 1E), indicating a change in immune activity in metastatic tumors and highlighting the observation of fewer
PTPN2+ infiltrating immune cells at this stage. Stage IV tumors emerge upon significant changes in the expression of various genes that not only enables tumor cell migration and metastasis formation, but also evasion of detection by the immune system. These changes not only affect gene expression in tumor cells, but likely also affect the activity and function of PTPN2, not only in the tumor but also in surrounding immune cells, specifically $\mathrm{T}$ cells. Thus, it is not surprising that expression of the analyzed factors STAT1, CXCL11, and GZMA, which are associated with immune cell infiltration and activation, shows an opposite correlation with PTPN2 in stage IV tumors.

Finally, we explored PTPN2 protein expression and localization of checkpoint molecules in primary CRC, liver metastasis, and melanoma. For primary CRC, we found that high PTPN2 expression in CRC tumor tissues correlated with low checkpoint molecule expression, similar to liver metastasis, in which low levels of PTPN2 expression within tumor tissues were associated with strong PD-1 and CTLA4 staining (Figure 1F and Supplemental Figure 1, A and B; supplemental material available online with this article; https://doi.org/10.1172/JCI140281DS1). On the contrary, we observed high levels of PTPN2 expression, which slightly positively correlated with expression of PD-1 in melanoma, showing that highly immunogenic tumors have a different PD-1 association with PTPN2 (Supplemental Figure 1A). Taken together, these patient studies demonstrate an increase in PTPN2 levels in immune cells in progressing CRC tumors and reveal that PTPN2 could be a mediator of decreased CRC immunogenicity.

Loss of PTPN2 in $T$ cells reduces tumor burden in the AOM/ DSS tumor model. Our results from patients suggest a crucial role of PTPN2 in immune cells during CRC development. To confirm this hypothesis, we investigated the role of PTPN2 in T cells in CRC mouse models. In a first approach, we applied the azoxymethane/dextran sodium sulfate (AOM/DSS) CRC model in $P t p n 2^{f / f l} C d 4^{\mathrm{Cret}+\text { - }}$ (referred to as $\Delta \mathrm{T}$ in the figures) mice lacking PTPN2 specifically in both $\mathrm{CD}^{+}$and $\mathrm{CD}^{+} \mathrm{T}$ cells $(22,23)$. $P t p n 2^{f l / f l}$ (WT) littermates not carrying the Cre construct were used as controls (Figure 2A).

In AOM/DSS-treated WT mice, we observed prominent tumor growth in the distal part of the colon when compared with $P t p n 2^{f l / f l} C d 4^{C r e+/-}$ littermate controls, whereas the controls showed no visual differences between genotypes (Figure $2 \mathrm{~B}$ ). The size and number of tumors were significantly reduced in $\mathrm{Ptpn} 2^{f / f l} \mathrm{Cd} 4^{\mathrm{Cre}+/-}$ mice compared with their WT littermate controls (Figure 2C). $\mathrm{H} \& \mathrm{E}$ staining of colon sections showed highly increased immune cell infiltration into tumor tissues in $P t p n 2^{f / / f l} C d 4^{C r e+/-}$ mice compared with WT littermates (Figure 2D), and IHC staining for CD3 revealed augmented accumulation of $\mathrm{T}$ cells into the tumors of these mice (Figure 2E).

RNA-Seq of colon tissues from water-treated (healthy tissue) and AOM/DSS-treated (nontumor and tumor tissue) WT and $P \operatorname{tpn} 2^{f / / f l} \mathrm{Cd} 4^{\mathrm{Cret} /-}$ mice revealed that AOM/DSS treatment in Ptpn $2^{f l / f l} C d 4^{C r e+/-}$ mice showed the highest change in the expression of genes involved in $\mathrm{CD}^{+} \mathrm{T}$ cell signaling, cytotoxicity, and infiltration, including STAT1, IFN- $\gamma$, granzyme family, checkpoint molecule, and chemokine genes (Figure $2 \mathrm{~F}$ and Supplemental Figure 2A). We performed quantitative PCR (qPCR) to confirm the significant changes in chemokine expression observed in the 
A

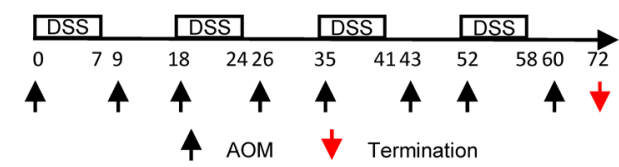

C

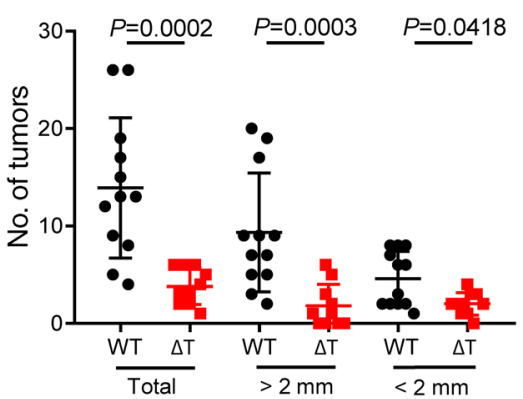

D

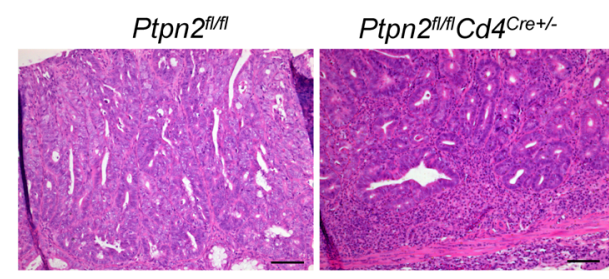

B
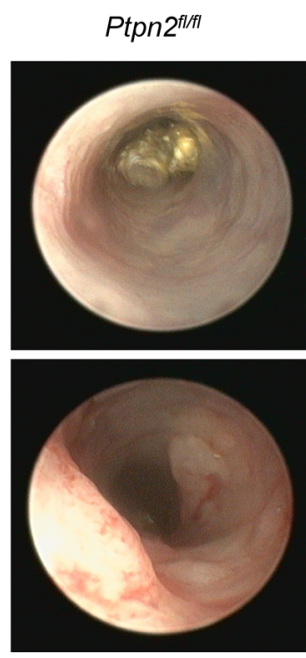

Ptpn $2^{\text {ff/fil }} \mathrm{Cd} 44^{\mathrm{Cre}+/-}$

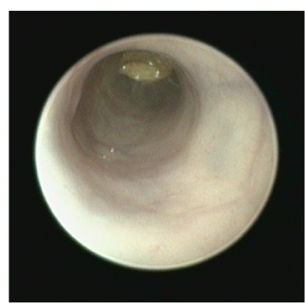

Untreated

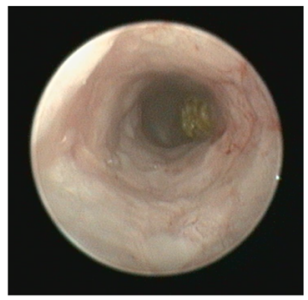

AOM/DSS
E

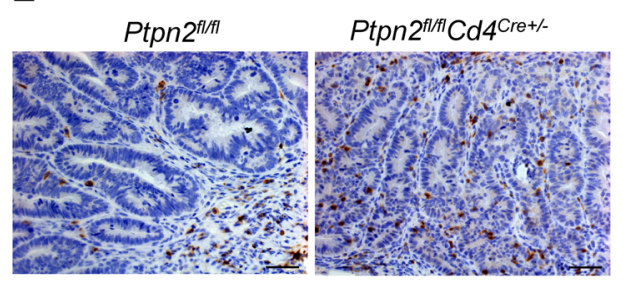

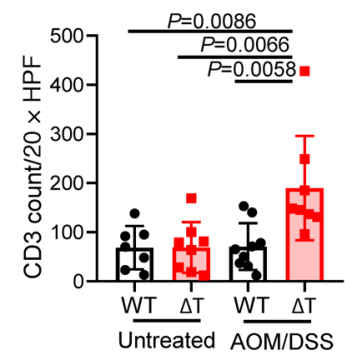

$\mathbf{F}$

T cell signalling

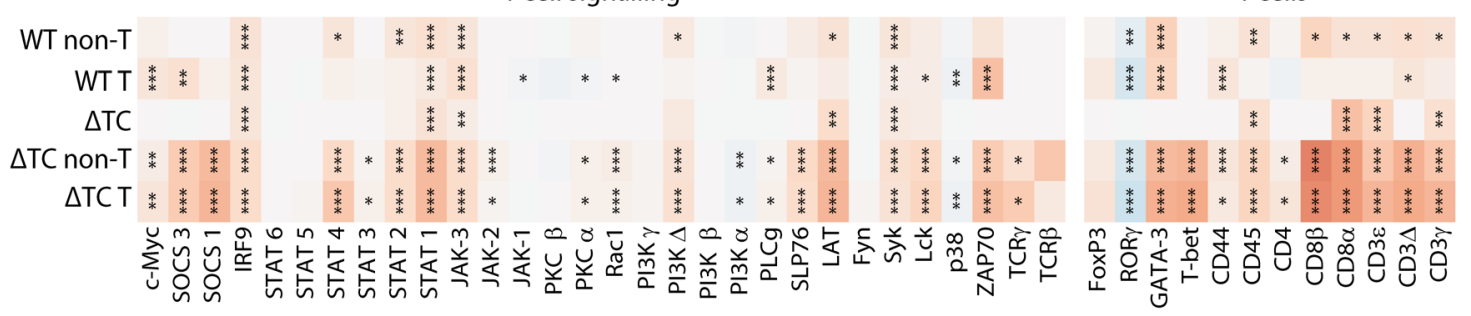

Checkpoint molecules

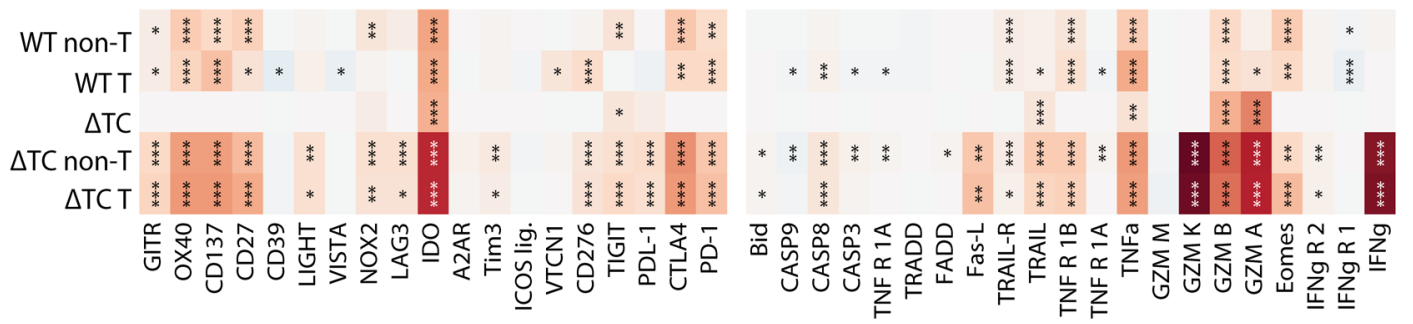

Costimulatory
molecules

Chemokines

Cytokines

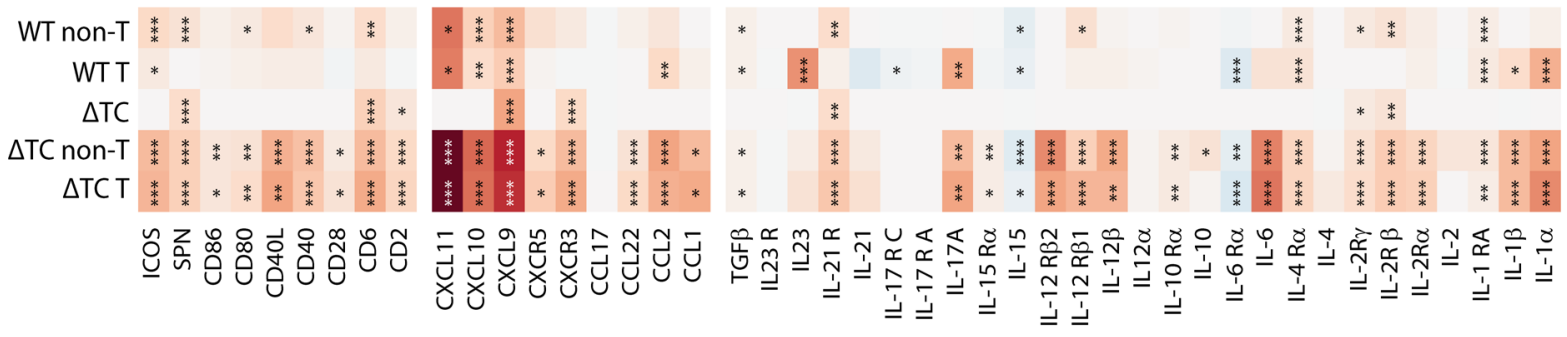

$\log _{2}$ fold change rel. to WT

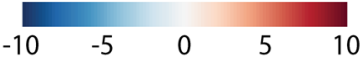


Figure 2. PTPN2 deletion in T cells leads to reduced tumor burden in colitis-associated tumors. Tumors were induced in Ptpn $2^{f / f l} C d 4^{\text {Cret/- }}$ mice $(\Delta T)$ $(n=10)$ and littermate control Ptpn $2^{f / / f l}$ mice (WT) $(n=12)$ using the AOM/ DSS model ( $n=2$ independent experiments). Mixed WT controls (Ptpn $2^{f / f l}$ $C d 4^{\text {Cre- }}$ and Ptpn $\left.2^{f / f f l} C d 11 c^{C r e-}\right)$ were used in this experiment. (A) Schematic overview of the experimental procedure. (B) Representative colonoscopy images from untreated and AOM/DSS-treated mice. (C) Quantification of the total number of tumors, stratified by tumor size. $P$ values were determined by 2-tailed Mann-Whitney $U$ test. (D) H\&E staining of tumor tissue from Ptpn $2^{f / / f l} \mathrm{Cd} 4^{\mathrm{Cr}+/ /}$ and Ptpn $2^{\mathrm{fl} / f l}$ mice. Scale bars: $100 \mu \mathrm{m}$. (E) Representative images and quantification of CD3 staining in Ptpn $2^{\mathrm{fl} / f l} \mathrm{Cd} 4^{\mathrm{Cr}+/-}$ and WT tumor tissue. RNA-Seq was performed on untreated, inflamed nontumor, and inflamed tumor tissue from WT and Ptpn $2^{f / / f l} C d 4^{C r e+/-}$ mice $(n=4$ mice each). Scale bars: $50 \mu \mathrm{m}$. $P$ values were determined by 1-way ANOVA with Tukey's multiple-comparison test. (F) Heatmap of mRNA expression levels of T cell-related genes from WT inflamed nontumor tissue (WT non-T), WT inflamed tumor tissue (WT T), Ptpn $2^{\text {fl/fl }} \mathrm{Cd} 4^{\text {(ret+/- }}$ untreated

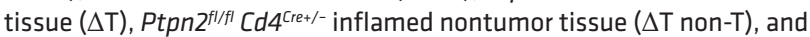
$P \operatorname{tpn} 2^{f / / f l} \mathrm{Cd}^{\mathrm{Cre+} /-}$ inflamed tumor tissue $(\Delta \mathrm{T} \mathrm{T})$ normalized to the expression levels in colon tissue from water-treated WT mice. False sign rate, ${ }^{*} P<$ $0.01,{ }^{* *} P<0.001$, and ${ }^{* *} P<0.0001$. Data represent the mean \pm SD.

RNA-Seq analyses (Supplemental Figure 2B). Similar to the data obtained by RNA-Seq, we found that CXCL9, CXL11, CXCR3, and CXCR5 chemokines were significantly upregulated in tumor tissue from $P t p n 2^{f l / f l} C d 4^{\mathrm{Cret} / \mathrm{-}}$ mice compared with expression levels in tissue from their littermate controls. Collectively, these findings indicate that PTPN2 deletion in T cells led to changes in the cytotoxic $\mathrm{T}$ cell compartment in the inflammation-dependent AOM/ DSS CRC model.

PTPN2 regulates the expression, activation, and tumor infiltration of $C D 8^{+}$cytotoxic $\mathrm{T}$ cells. To further investigate the alterations in $\mathrm{T}$ cell function/activation caused by PTPN2 deletion, we examined $\mathrm{CD}^{+}$and $\mathrm{CD}^{+} \mathrm{T}$ cells in detail. IHC and flow cytometric analyses of $\mathrm{CD}^{+} \mathrm{T}$ cells in healthy colon, inflamed nontumor, and tumor tissues did not reveal significant differences in the frequencies of $\mathrm{CD} 4^{+} \mathrm{T}$ cells between Ptpn $2^{f l / f l} \mathrm{Cd} 4^{\mathrm{Cret} /-}$ and WT mice (Figure $3 \mathrm{~A}$ ). Nevertheless, effector/memory CD $4^{+} \mathrm{T}$ cells were increased in the spleen and mesenteric lymph nodes (mLNs) of Ptpn $2^{f / f l} \mathrm{Cd} 4^{\mathrm{Cret} /-}$ mice, independent of the treatment (Figure 3B). However, no signs of microscopic inflammation or morphologic changes were detectable in the colon of unchallenged Ptpn $2^{f l / f l} C d 4^{\mathrm{Cre+}+-}$ mice. Flow cytometry showed a clear increase in Th1 $\left(\mathrm{CD} 4^{+} \mathrm{IFN}-\gamma^{+}\right)$cells, while Th2 cells $\left(\mathrm{CD} 4^{+} \mathrm{IL}-4^{+}\right)$, Th17 cells $\left(\mathrm{CD} 4^{+} \mathrm{IL}-17^{+}\right)$, and Tregs $\left(\mathrm{CD}^{+} \mathrm{FoxP}^{+}\right)$showed no significant changes between Ptpn $2^{f / f l}$ $C d 4^{\mathrm{Cret} /-}$ mice and their WT littermate controls (Figure $3 \mathrm{C}$ and Supplemental Figure 3A).

Of note, we detected increased infiltration and frequency of $\mathrm{CD}^{+} \mathrm{T}$ cells in inflamed nontumor and tumor tissues from Ptpn $2^{f / f l}$ $C d 4^{\text {Cret/- }}$ mice by IHC and flow cytometry (Figure 3D). This increase in $\mathrm{CD}^{+} \mathrm{T}$ cell infiltration is well in line with the upregulation of chemokines and chemokine receptors observed in the tumor tissue. A predominant fraction of $\mathrm{CD} 8^{+} \mathrm{T}$ cells was activated effector/ memory $\left(\mathrm{CD}^{+} \mathrm{CD}^{+} 4^{+} \mathrm{CD} 62 \mathrm{~L}^{-}\right) \mathrm{T}$ cells in the spleen and mLNs of both water-treated control and AOM/DSS-treated mice (Figure 3E). Although comparable frequencies of PTPN2-deficient $\mathrm{CD}^{+} \mathrm{T}$ cells were activated in water- and AOM/DSS-treated mice, the frequency of granzyme B-positive cells was clearly increased specifically in tumor tissue, an effect completely absent in WT mice (Figure 3F and
Supplemental Figure 3, A-C). Similar to $\mathrm{CD}^{+}{ }^{+} \mathrm{T}$ cells, PTPN2-deficient $\mathrm{CD}^{+} \mathrm{T}$ cells expressed more IFN- $\gamma$ than did those from WT mice, but the frequencies did not differ between healthy tissue, inflamed (nontumor) tissue, and tumor tissue (Figure 3F).

In WT mice, the frequency of $\mathrm{CD} 4^{+} \mathrm{PD}-1^{+}$, but not $\mathrm{CD} 8^{+} \mathrm{PD}-1^{+}$, $\mathrm{T}$ cells was significantly increased in nontumor and tumor tissue when compared with frequencies in healthy tissue (Figure $3 F$ ). In contrast, the frequency of $\mathrm{CD} 4^{+} \mathrm{PD}-1^{+}$cells did not differ between tissues from water- and AOM/DSS-treated Ptpn $2^{f / / f l} \mathrm{Cd} 4^{\mathrm{Cret}+-}$ mice and was overall significantly lower than that in WT littermates (Figure 3F). Nevertheless, we observed a clear increase in PD-1 expression on $\mathrm{Ptpn} 2^{f / / f l} \mathrm{Cd} 4^{\mathrm{Cret} /-} \mathrm{CD}^{+} \mathrm{T}$ cells in inflamed nontumor colon and tumor tissues compared with healthy colon tissues, but its expression was still lower than in WT mice (Figure 3F).

PTPN2 T cell deficiency reduces tumor burden independently of inflammation. We next assessed the tumor burden in inflammation-independent models of CRC, namely the orthotopic MC38 cell cecal injection model and the genetic APCmin mouse model.

Remarkably, using the cecal injection model, approximately $60 \%$ of $P \operatorname{tpn} 2^{f / / f l} C d 4^{C r e+/-}$ mice completely cleared the tumors, and the remaining tumors were relatively small, whereas WT mice showed a failure of tumor control (Figure 4, A and B). IHC and flow cytometric analyses of $\mathrm{CD} 8^{+} \mathrm{T}$ cells showed enhanced infiltration of $\mathrm{CD}^{+} \mathrm{T}$ cells into tumors in Ptpn $2^{f / f l} \mathrm{Cd} 4^{\mathrm{Cre}+-}$ mice compared with WT littermate controls (Figure 4C). Again, we observed increased expression of chemokines in tumors from Ptpn $2^{f / f l}$ $C d 4^{\mathrm{Cret} /-}$ mice, which further supports the idea of increased $\mathrm{CD} 8^{+}$ $\mathrm{T}$ cells infiltration into the tumor as a mechanism for reduced tumor burden (Supplemental Figure 4A).

We observed a comparable increase in systemic $\mathrm{CD}^{+} \mathrm{T}$ cell activation upon loss of PTPN2 in both the AOM/DSS and cecal injection tumor models, suggesting that initial inflammation was not required for $\mathrm{T}$ cell activation or tumor clearance (Figure 4D). Furthermore, we found similar expression levels of cytotoxicity markers in both tumor models, including increased granzyme B and IFN- $\gamma$ expression in tumor tissues from Ptpn $2^{f / / f l} \mathrm{Cd} 4^{\mathrm{Cre+} /-}$ mice compared with expression in WT littermate controls (Figure 4E). Notably, in the cecal injection model, PD-1 expression on $\mathrm{CD}^{+}$ $\mathrm{T}$ cells was significantly increased in $\mathrm{Ptpn} 2^{f / f l} \mathrm{Cd} 4^{\mathrm{Cret}+-}$ mice compared with expression in WT control mice (Figure 4E).

In the genetic APCmin model of CRC, Ptpn $2^{f / / f l} \mathrm{Cd} 4^{\mathrm{Cret} /-} \mathrm{APC}$ min mice showed an extensive reduction of tumor burden in the small intestine when compared with Ptpn $2^{f / f l} A P C m i n$ littermates (Figure $4 \mathrm{~F}$ ), and Ptpn $2^{f / f l} \mathrm{Cd} 4^{\mathrm{Cret} /-} \mathrm{APCmin}$ mice had healthy spleens, whereas Ptpn $2^{f / f l} A P C m i n$ mice had spleens that were significantly heavier (Figure 4F) and an unhealthy, pale color.

Finally, to validate whether PTPN2 deletion in T cells exerts not only local effects in the colon, but is also relevant for systemic tumors, we next applied the s.c. injection model using MC38 CRC and B16 melanoma cells. Consistent with our findings with in situ tumors, we observed slower tumor growth and reduced size and weight in Ptpn $2^{f / f l} \mathrm{Cd} 4^{\mathrm{Cret}+-}$ mice compared with WT mice, regardless of the cancer cell type, i.e., MC38 or B16 (Figure 5, A and B, and Supplemental Figure 5, A and B).

PTPN2-deficient $C D 8^{+} T$ cells are the key mediators of antitumor efficacy. Given the prominent changes in $\mathrm{CD}^{+} \mathrm{T}$ cells revealed in all our data, $\mathrm{CD} 8^{+} \mathrm{T}$ cell depletion in the MC38 s.c. injection mod- 
A
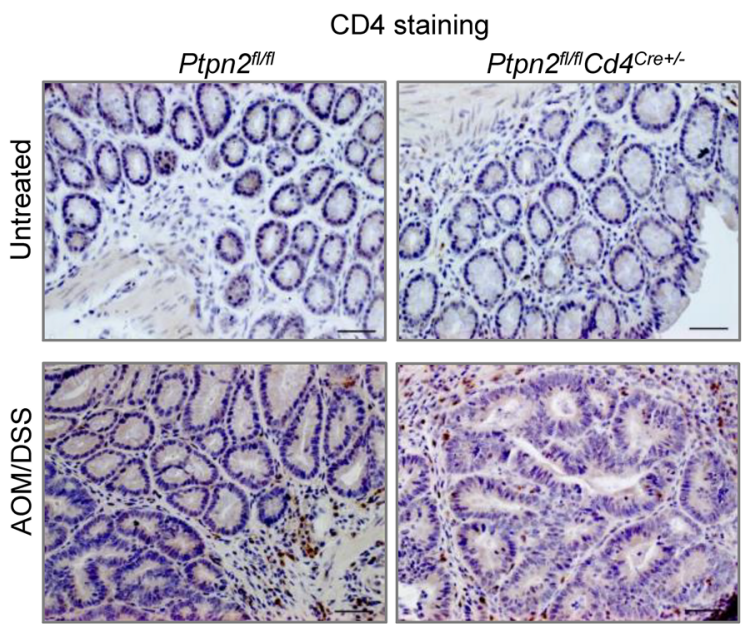

C
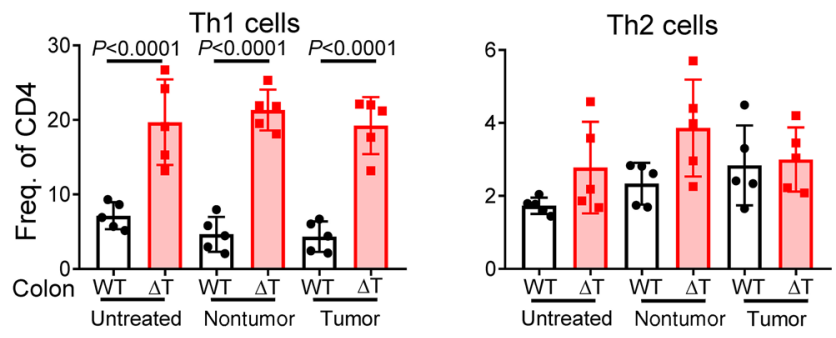

D
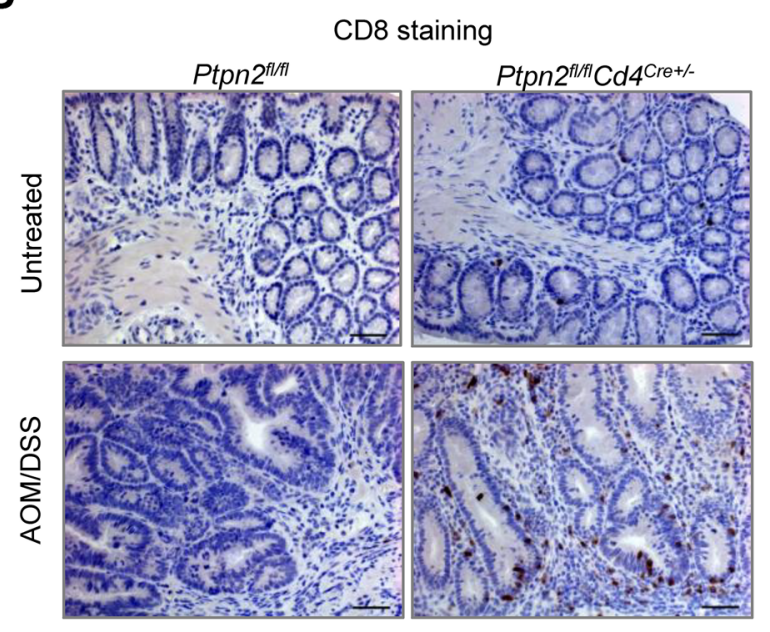

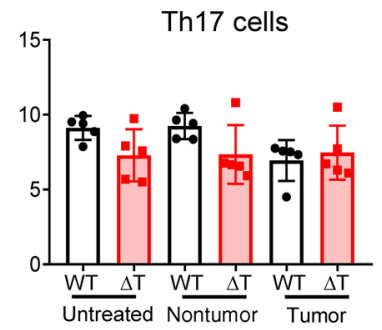

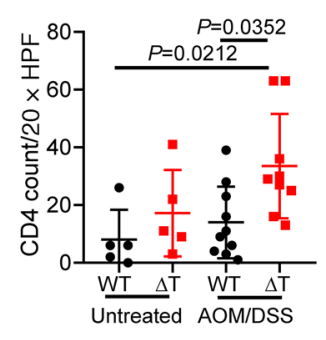

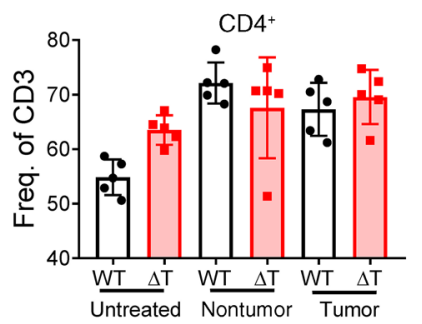

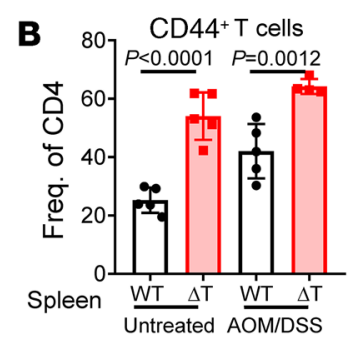

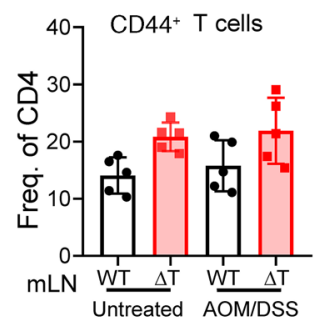

Treg cells

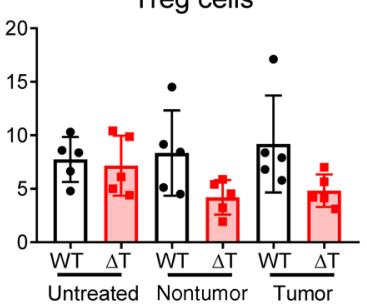

E
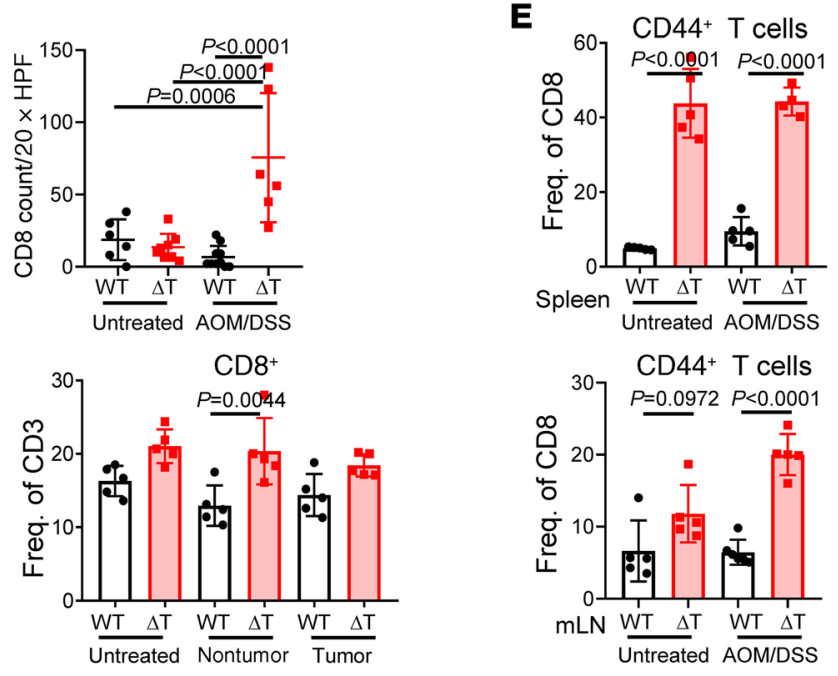

$\mathbf{F}$
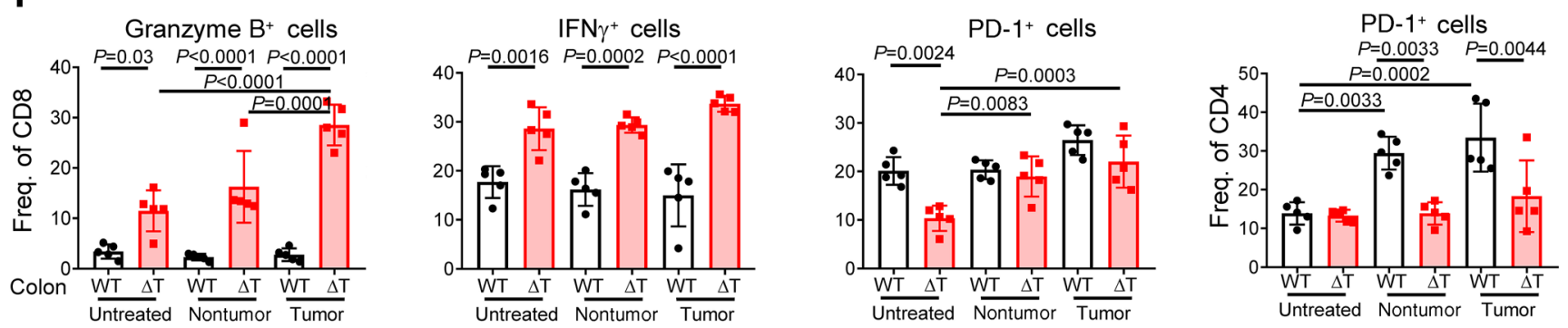
Figure 3. PTPN2 deletion in T cells promotes $\mathrm{T}$ cell activation in tumor tissue. Tumors were induced as described in Figure 2. (A) Representative images and quantification of CD4 IHC staining and flow cytometric analysis of WT and $P \operatorname{tpn} 2^{f / / f l} \mathrm{Cd} 4^{\mathrm{Cr}+\mathrm{r} / \mathrm{C}}$ untreated and tumor tissue. Flow cytometric analysis of untreated colon tissue, inflamed nontumor tissue, and inflamed tumor tissue ( $n=5$ mice in each group). Scale bars: $50 \mu \mathrm{m}$. $P$ values were determined by 1-way ANOVA with Tukey's multiple-comparison test. (B and C) Frequencies of $C D 4^{+} T$ cells, effector/memory CD4 ${ }^{+} C D 44^{+} T$ cells (spleen and $\mathrm{mLNs}$ ), and Th1 cells (CD4+IFN- $\left.\gamma^{+}\right)$, Th2 cells (CD4+IL-4+), Th17 cells (CD4+IL-17+), and Tregs (CD4+FoxP3 ${ }^{+}$) (colon). $P$ values were determined by 1-way ANOVA with Tukey's multiple-comparison test. (D) Localization and quantification of $\mathrm{CD}^{+}$cells in

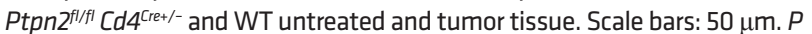
values were determined by 1-way ANOVA with Tukey's multiple-comparison test. (E) Frequencies of effector/memory CD8 ${ }^{+} \mathrm{CD} 44^{+} T$ cells (spleen and $\mathrm{mLNs}$ ). $P$ values were determined by 1-way ANOVA with Tukey's multiple-comparison test. (F) Frequencies of $\mathrm{CD}^{+}$granzyme $\mathrm{B}^{+}, \mathrm{CD}^{+} \mathrm{IFN}-\gamma^{+}$, and the checkpoint molecule PD-1 on CD4 ${ }^{+}$and CD8 ${ }^{+}$T cells. $P$ values were determined by 1-way ANOVA with Tukey's multiple-comparison test. Data represent the mean \pm SD. Freq., frequency.

el abrogated the protective effect of PTPN2 deficiency, resulting in similar tumor growth and terminal weight in $\operatorname{Ptpn} 2^{f / f l} \mathrm{Cd} 4^{\mathrm{Cre+}+-}$ mice and WT littermates (Figure 5, C and D).

IHC staining and flow cytometric analysis revealed highly increased infiltration of PTPN2-deficient $\mathrm{CD}^{+} \mathrm{T}$ cells into the tumor in isotype-treated control mice, whereas $\mathrm{CD} 8^{+} \mathrm{T}$ cells were successfully depleted in both mouse lines (Figure 5E). Of note, we observed an inverse correlation between tumor weight and CD8 ${ }^{+}$ $\mathrm{T}$ cell frequency in the tumor tissues from $\mathrm{Ptpn} 2^{\mathrm{fl} / \mathrm{fl}} \mathrm{Cd} 4^{\mathrm{Cret} /-}$ mice, while in WT mice, such a correlation was not detectable (Figure $5 \mathrm{~F}$ ).

In contrast to the drastic effects of CD8 depletion, $\mathrm{CD} 4^{+} \mathrm{T}$ cell depletion did not affect the tumor suppression capacity in Ptpn $2^{f l / f l} C d 4^{C r e+/-}$ mice. Although tumor growth was slightly suppressed upon $\mathrm{CD}^{+} \mathrm{T}$ cell depletion in WT mice (Figure $5, \mathrm{G}$ and $\mathrm{H}$, and Supplemental Figure 6A), which might have resulted from the depletion of Tregs, we did not further investigate this in this study. Flow cytometric analysis revealed complete $\mathrm{CD} 4^{+} \mathrm{T}$ cell depletion in the spleen and a reduction of approximately $80 \%$ in tumor tissue (Supplemental Figure 6B). The expression of granzyme B and IFN- $\gamma$ was significantly higher in Ptpn $2^{f / / f l} \mathrm{Cd} 4^{\mathrm{Cret} /-}$ mice when compared with expression in WT mice with or without CD4 depletion (Supplemental Figure 6C). Thus, CD $4^{+}$T cells were not required for tumor suppression in $\mathrm{Ptpn} 2^{f / f l} \mathrm{Cd} 4^{\mathrm{Cre+} /-}$ mice.

To further investigate the antitumor potential of PTPN2deficient $\mathrm{CD}^{+} \mathrm{T}$ cells, we injected MC38 cells into Rag2-deficient mice, followed by adoptive transfer of either WT or PTPN2deficient $\mathrm{CD}^{+} \mathrm{T}$ cells on day 6 (Figure 6A). Remarkably, tumor growth was significantly slower in mice that received $\mathrm{CD}^{+} \mathrm{T}$ cells from $P t p n 2^{f l / f l} C d 4^{C r e+/-}$ mice, further demonstrating the prominent role of $\mathrm{CD}^{+} \mathrm{T}$ cells in reducing tumor burden in $\mathrm{Ptpn} 2^{\mathrm{fl} / \mathrm{fl}} \mathrm{Cd} 4^{\mathrm{Cre+}+-}$ mice (Figure 6B).

We confirmed the increased antitumor capacity of PTPN2deficient $\mathrm{T}$ cells in an in vitro killing assay, in which we incubated MC38 and MC38-OVA-expressing cells with Ptpn $2^{f / / f l} O T-I$ or

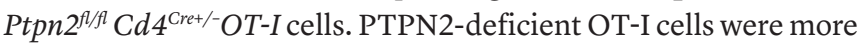
efficient at antigen-specific tumor killing when compared with PTPN2-proficient OT-I cells (Figure 6C).

PTPN2 deletion in DCs leads to reduced tumor burden in both $A O M / D S S$ and orthotopic injection models. In light of our findings in mice lacking PTPN2 in T cells, we investigated whether deletion of PTPN2 in antigen-presenting cells, specifically DCs, also affects tumor development in our CRC models. Of note, our previous work showed a significant tumor reduction in mice lacking PTPN2 in myeloid cells (19), but the effect of PTPN2 depletion in DCs on CRC development is still unknown.

To this aim, we first subjected Ptpn $2^{f / f l} C d 11 C^{C r e+/-}$ mice and their WT littermate controls $\left(P t p n 2^{f / f f}\right)$ to the AOM/DSS model and observed a significant reduction in tumor load and enhanced $C D 8^{+} \mathrm{T}$ cell infiltration in $\mathrm{Ptpn} 2^{f l / f l} \mathrm{Cd} d 1 c^{\mathrm{Cret} /-}$ mice compared with their littermates (Figure 7, A-C). Similar to Ptpn $2^{f / f l} \mathrm{Cd} 4^{\mathrm{Cre}+-}$ mice, we observed enhanced levels of effector/memory T cells in Ptpn $2^{f / f l} \mathrm{Cd} 11 c^{\mathrm{Cret} /-}$ mice (Figure 7C), suggesting that PTPN2-deficient DCs promote $\mathrm{T}$ cell activation. This finding is well in line with a recently published study from our group (24).

Next, we applied the orthotopic MC38 cecal injection model of CRC in Ptpn $2^{f / / f l} C d 11 c^{C r e+/-}$ and Ptpn $2^{f / f l}$ mice and found cellular and pathologic phenotypes similar to those observed in the AOM/ DSS model, which, again, mirrored the situation in the Ptpn $2^{f / f l}$ $C d 4^{\text {Cret-- }}$ tumor models. We found a considerably reduced tumor load (Figure 7D), changes in tumor morphology (Figure 7E), and significantly increased infiltration, activation, and cytotoxicity of $\mathrm{CD}^{+} \mathrm{T}$ cells (Figure 7, F-H). Thus, we hypothesize that in Ptpn $2^{f / f l}$ $C d 11 c^{C r e+/-}$ mice, activated T cells, as in Ptpn $2^{f / / f l} C d 4^{\text {Cret/- }}$ mice, might mediate the reduction in tumor load.

PTPN2 deletion renders tumors more susceptible to anti-PD-1 treatment. To investigate whether PTPN2 deletion makes T cells more susceptible to immunotherapy, we injected MC38 tumor cells s.c. into WT and Ptpn $2^{f / / f l} C d 4^{\mathrm{Cret}+-}$ mice and administered anti-PD-1 antibody on days 9, 12, and 15, when large (100-200 $\mathrm{mm}^{3}$ ) tumor nodules had formed (Figure 8A). Already in PTPN2-deficient mice receiving the $\operatorname{IgG}$ isotype control, we observed reduced tumor growth, but, strikingly, almost all $\mathrm{Ptpn} 2^{f / / f l} \mathrm{Cd} 4^{\mathrm{Cre+}+-}$ mice treated with anti-PD-1 cleared the tumors by day 20 (Figure 8, A and B). This additive effect on tumor reduction suggests that PTPN2 deletion and anti-PD-1 therapy mediate tumor reduction through distinct mechanisms. In contrast, all WT mice reached the termination criteria because of tumor size, with or without anti-PD-1 treatment (Figure 8, A and B). Additionally, tumor morphology was altered in anti-PD-1-treated Ptpn $2^{f / / f l} \mathrm{Cd} 4^{\mathrm{Cret} /-}$ mice, as evidenced by reduced cancer cell density and patches of immune cell infiltrates (Figure $8 C$ ). Anti-PD-1 treatment promoted $\mathrm{CD}^{+} \mathrm{T}$ cell infiltration in PTPN2-deficient mice, but not in WT mice (Figure 8D), indicating that PTPN2 depletion in T cells renders tumors more susceptible to checkpoint inhibitor treatment.

We further noted that the frequency of $\mathrm{CD} 8^{+} \mathrm{PD}-1^{+}$cells was significantly increased in IgG isotype-treated Ptpn $2^{f / f l} \mathrm{Cd} 4^{\mathrm{Cre}+/}$ mice when compared with frequencies in WT controls (Figure 8E). Depletion of PD-1 was successful in both WT and Ptpn $2^{f / f l} \mathrm{Cd} 4^{\mathrm{Cret}+-}$ mice and evident in $\mathrm{CD} 8^{+} \mathrm{T}$ cells, yet the anti-PD-1 therapy had much stronger effects on tumor load in the PTPN2-deficient mice. Last, checkpoint blockade did not change the activation or cytotoxicity of T cells in WT mice, whereas Ptpn $2^{f / f l} \mathrm{Cd} 4^{\mathrm{Cret} / \mathrm{-}}$ mice had significantly higher frequencies of cytotoxic and activated $\mathrm{T}$ cells upon anti-PD-1 therapy (Figure 8E).

Increased immune memory formation in Ptpn $2^{f / f l} \mathrm{Cd} 4^{\mathrm{Cre+}+-}$ mice. To determine whether PTPN2 deletion affects tumor-specific 


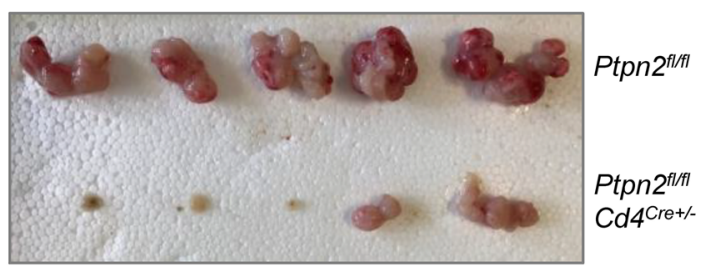

B
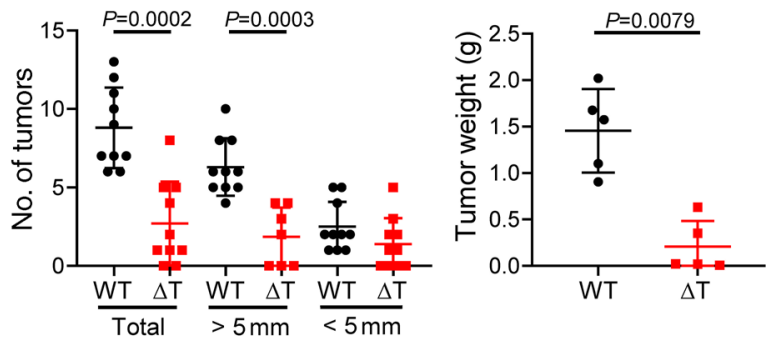

C

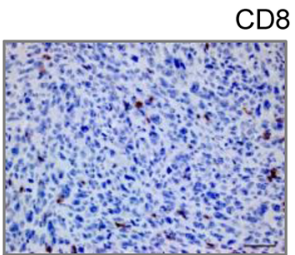

Ptpn2 $2^{\text {tht }}$
CD8 staining

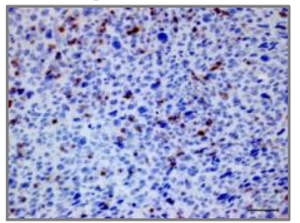

Ptpn $2^{\text {fiffil }} \mathrm{Cd} 44^{\mathrm{Cret} / 1}$
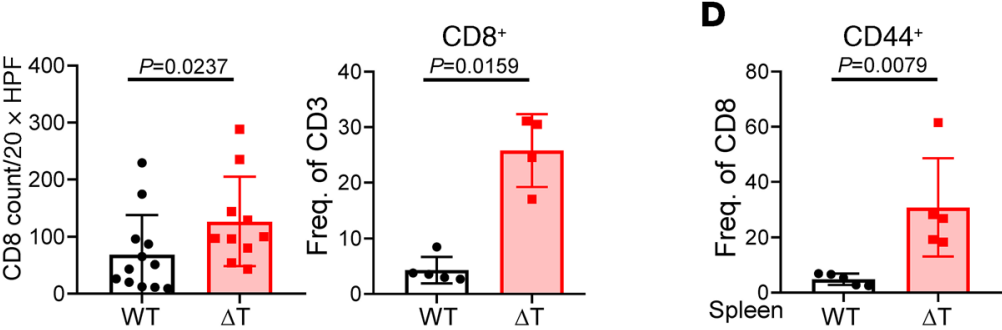

E

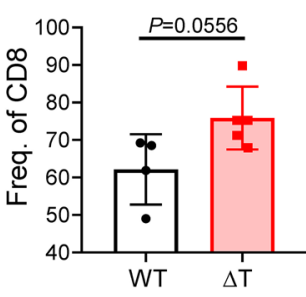

IFN- $\gamma^{+}$

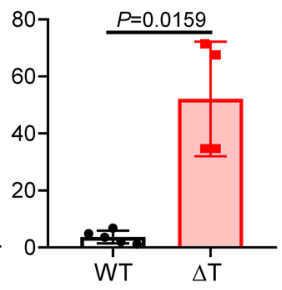

PD-1+

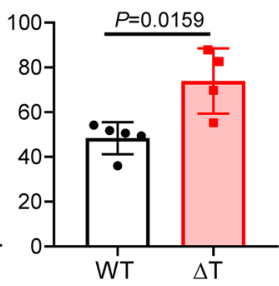

$\mathbf{F}$

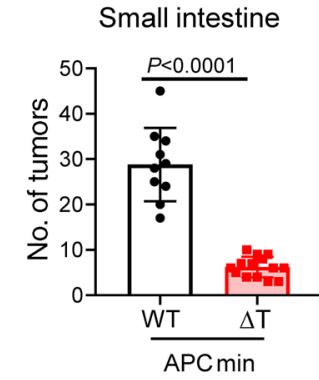

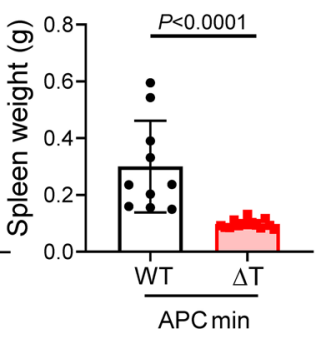

Figure 4. Reduced tumor burden in orthotopic tumor injection and APCmin tumor models upon PTPN2 deletion in T cells. MC38 tumor cells ( 300,000

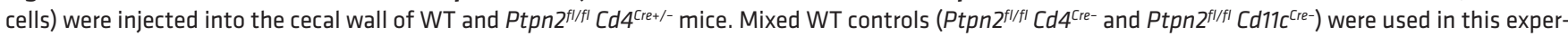
iment, therefore, WT quantification in $\mathbf{C}$ is the same as the WT quantification in Figure 7F. (A) Representative gross image of cecal tumors from WT and Ptpn $2^{f / f f} \mathrm{Cd} 4^{\mathrm{Cr} r+} /-$ mice $(n=5$ mice). (B) Total number of tumors and stratification according to size ( $n=10$ mice; $n=2$ independent experiments) and tumor weight ( $n=5$ mice). $P$ values were determined by 2 -tailed Mann-Whitney $U$ test. (C) Representative images and quantification of IHC staining and flow cytometric analysis of CD8 staining of Ptpn $2^{f / / f l}$ and Ptpn $2^{f l / f l} C d 4^{C r+t /-}$ tumor tissue. Scale bars: $50 \mu \mathrm{m}$. $P$ values were determined by 2-tailed Mann-Whitney $U$ test. Frequencies of effector/memory CD8 ${ }^{+} C D 44^{+} T$ cells in spleen (D) and of granzyme B, IFN- $\gamma$, and PD- 1 in tumor (E). $P$ values were determined by 2-tailed Mann-Whitney $U$ test. (F) Number of tumors in the small intestine and spleen weights for Ptpn $2^{f / f l} A P C m i n$ and Ptpn $2^{f / f l} C d 4^{C r e+/-} A P C m i n$ mice. $P$ values were determined by 2-tailed Mann-Whitney $U$ test. Data represent the mean \pm SD.

immune cell memory, we rechallenged $\operatorname{Ptpn} 2^{f / f /} \mathrm{Cd} 4^{\mathrm{Cret} / \text { - }}$ mice with a second injection of MC38 cells after they had cleared primary MC38 tumors following anti-PD-1 therapy. To compare primary and memory responses, we also injected MC38 cells into treatment-naive $P \operatorname{tpn} 2^{f / f l} C d 4^{C r e+/}$ mice (Figure $8 \mathrm{~F}$ ). Mice that were rechallenged with MC38 cells developed smaller tumors than did mice that received primary injections, and all rechallenged mice, but only few mice that received primary injections, cleared the tumors (Figure 8F). Thus, PTPN2 deficiency not only promoted tumor clearance in synergy with anti-PD-1 treatment, but also induced memory responses that mediated spontaneous tumor clearance upon rechallenge.

\section{Discussion}

In this study, we demonstrate that immune cell-specific PTPN2 plays an important role in the development of human CRC. Using a large collection of tissue samples from CRC with CRC, we show that PTPN2 protein expression and activity were highly elevated in human CRC tissue, with a marked increase in immune cells, and that high PTPN2 gene expression correlated with reduced $\mathrm{T}$ cell activity, recruitment, and cytotoxicity. Using well-defined mouse models, we verified that PTPN2 expression in T cells and DCs dampened antitumor immune responses and that PTPN2 deficiency led to significantly reduced tumor burden in vivo. Recent reports showed that PTPN2 silencing in T cells led to reduced tumor load in mice upon s.c. injection of MC38 and B16 cells and in mammary tumor mouse models $(12,13)$. While these studies showed altered $\mathrm{T}$ cell differentiation and enhanced antitumor response upon T cell-specific loss of PTPN2, they did not investigate human CRC tissues, nor did they examine whether cells of the in situ tumor microenvironment - which are critical for determining the therapeutic response - affect CRC tumor growth in vivo. In contrast, our study explored the potential of PTPN2 as a therapeutic target in different CRC models that adequately represent the tumor environment and development observed in patients with CRC.

Since in the majority of patients with CRC, low immunogenicity of the tumors prevents response to immunotherapies ( 7 , 
A

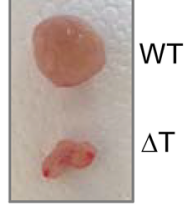

B

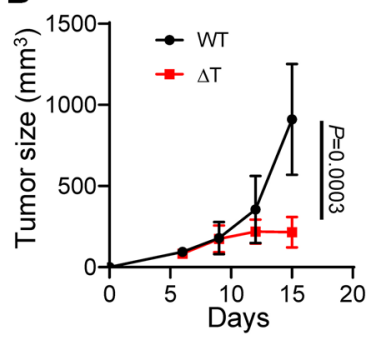

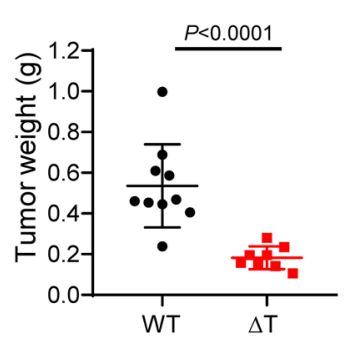
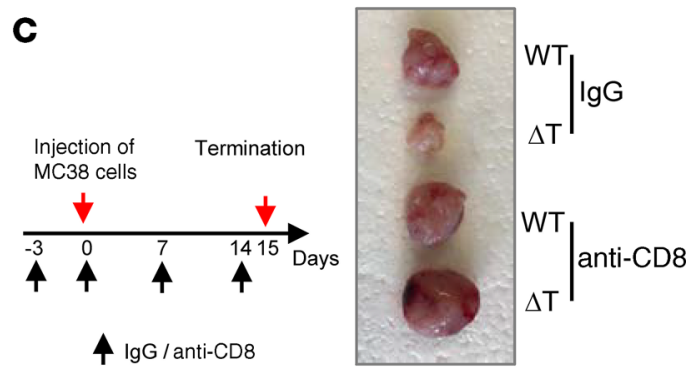

D
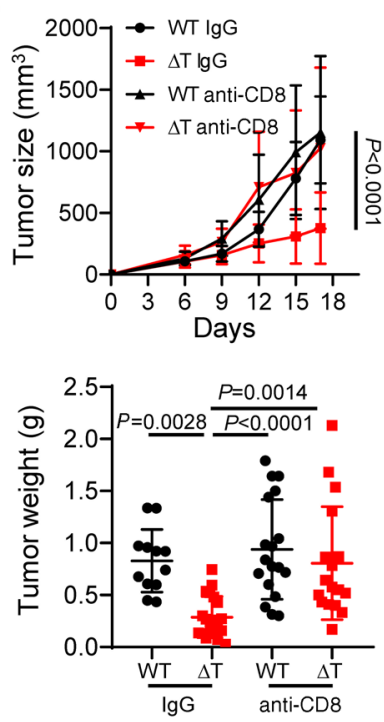

$\mathbf{F}$

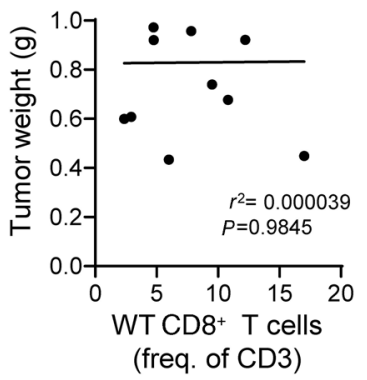

$\mathbf{E}$
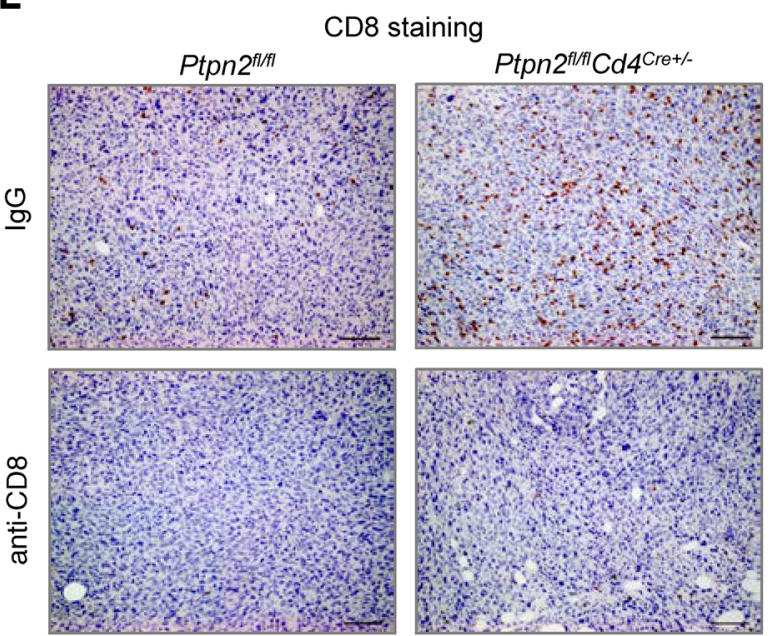

G

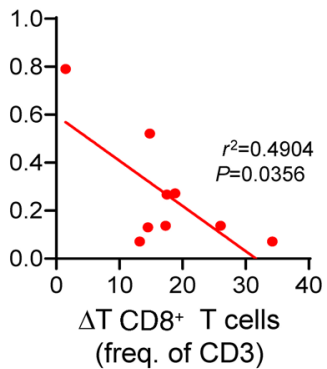

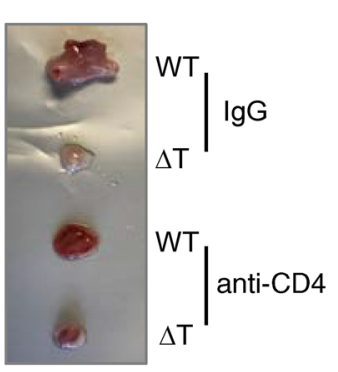
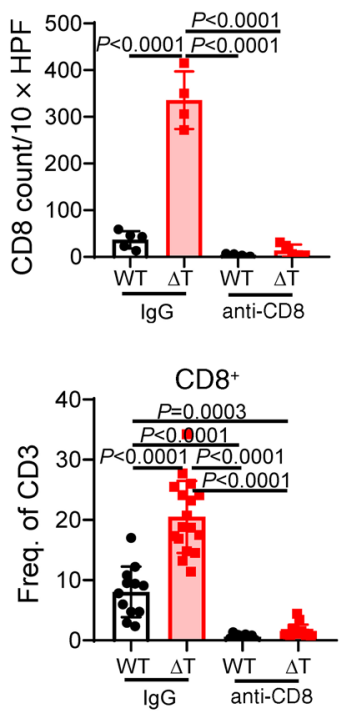

H

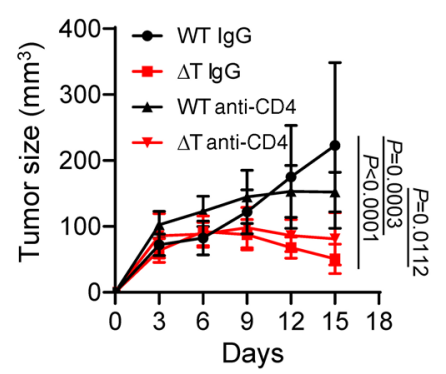

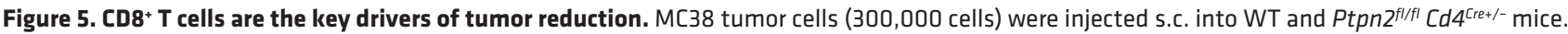
(A) Representative image of MC38-induced tumors from WT and Ptpn $2^{f / f l}$ Cd4 ${ }^{\mathrm{Cre+} /-}$ mice. (B) MC38-induced tumor development ( $n=5$ WT mice and 10 tumors; $n=4$ Ptpn $2^{f / f l} C d 4^{\text {Cre+/- }}$ mice and 8 tumors) over time and tumor weights on the last day of the experiment. $P$ values were determined by 2 -tailed Mann-Whitney $U$ test. (C) Scheme of CD8+ T cell depletion and image of representative tumors from IgG isotype control- and anti-CD8-treated WT and Ptpn $2^{f / / f l} C d 4^{\text {Cre+/- }}$ mice. (D) Tumor sizes and weights ( $n=6$ WT IgG-treated mice and 12 tumors; $n=10$ mice and 20 tumors for lgG-treated Ptpn $2^{f / f l}$ Cd $4^{\text {Cre+ } /-}$ mice, anti-CD8-treated WT mice, and anti-CD8-treated Ptpn $2^{f / f l}$ Cd4 ${ }^{\text {Cre+/- }}$ mice; $n=2$ independent experiments). $P$ values were determined by 1-way ANOVA with Tukey's multiple-comparison test. (E) Representative images and quantification of IHC and flow cytometric CD8 staining of WT and Ptpn $2^{\text {flffl}}$ $C d 4^{\text {Cre+/- }}$ mouse tumor tissue from IgG control- and anti-CD8-treated groups. Scale bars: $50 \mu m$. $P$ values were determined by 1-way ANOVA with Tukey's multiple-comparison test. (F) Inverse correlation of tumor weight and frequency of CD8 ${ }^{+}$T cells in tumor tissue from WT and $P$ tpn $2^{f / f l}$ Cd4 ${ }^{\text {Cre+/- }}$ mice. $P$ and $R^{2}$ values were determined by linear regression analysis. (G) Representative gross image of MC38 tumors from IgG isotype control- and anti-CD4-treated WT and Ptpn $2^{f / f l} C d 4^{\text {Cre+/- }}$ mice. (H) Tumor development ( $n=5$ mice and 10 tumors per group). $P$ values were determined by 1 -way ANOVA with Tukey's multiple-comparison test. Data represent the mean $\pm \mathrm{SD}$.

$8,25,26)$, therapeutic approaches that promote the immunogenic potential would result in improved treatment success. Our data from primary and metastatic human CRC tissues indicate a strong correlation between local PTPN2 expression and a reduced immune response. Thus, our observations in patients indicate that PTPN2 is an important target for CRC therapy.
PTPN2 is a known antiinflammatory protein that dephosphorylates several inflammatory mediators and prevents tissue inflammation (15-17,21). PTPN2 deficiency in mice has previously been shown to affect pathologic immune responses as well as the development of $\mathrm{T}$ cells and $\mathrm{B}$ cells, to mediate hypersensitivity of macrophages, and to enhance the production of proinflammatory cytokines such 
A

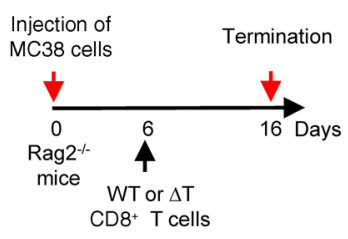

B

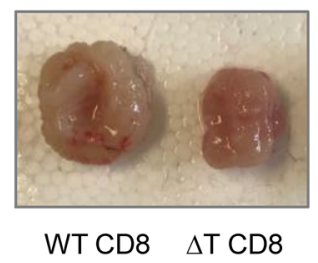

Early apoptotic
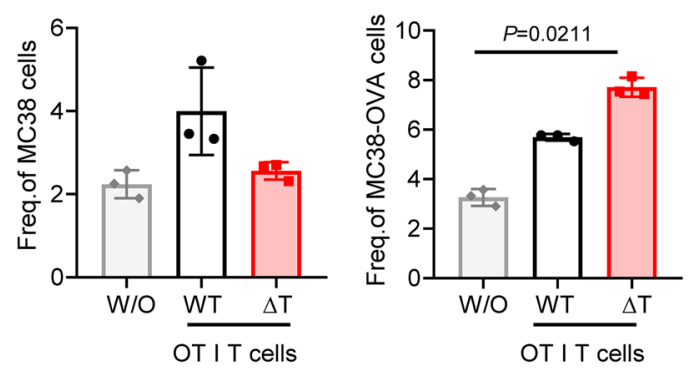

C
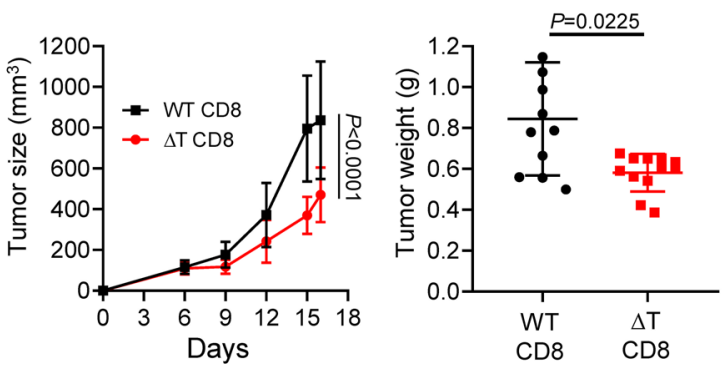

Late apoptotic

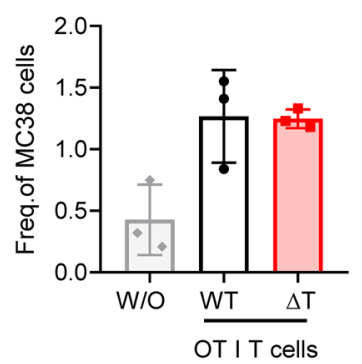

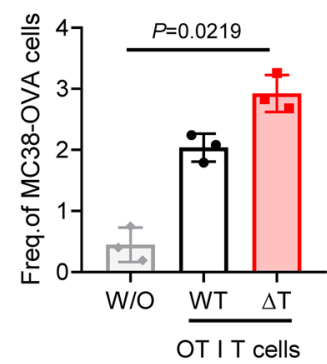

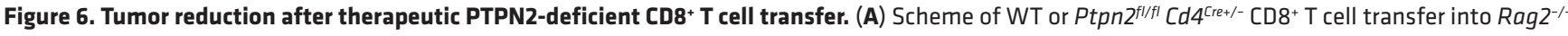
mice. (B) Representative image, development curve, and weights of MC38 tumors from mice without T cell transfer ( $n=6$ mice and 12 tumors), or with either WT CD8 ${ }^{+}$T cell ( $n=5$ mice and 10 tumors) or Ptpn2 $2^{f / f l} C d 4^{\text {cret/- }} \operatorname{CD8}^{+}$( $n=6$ mice/ 12 tumors) T cell transfer. $P$ values were determined by 2-tailed Mann-Whitney $U$ test. (C) In vitro killing assay of MC38 and MC38-OVA cells by WT and Ptpn $2^{f / f l}$ Cd4 $4^{\text {Cret/- }}$ OT-I T cells. Graphs represent early (annexin $\mathrm{V}^{+}$zombieNIR-) and late (annexin $\mathrm{V}^{+}$zombieNIR+) apoptotic cells. $P$ values were determined by 1-way ANOVA with Tukey's multiple-comparison test. Data represent the mean \pm SD. W/O, without addition of T cells.

as IFN- $\gamma$, TNF, and IL-1 $\beta(18,19,21)$. We previously showed that conditional PTPN2 KO (cKO) in T cells promotes Th1 and Th17 differentiation and results in increased IFN- $\gamma$ and IL-17 production in colitis models (17). Furthermore, $\mathrm{T}$ cell cKO has been shown to promote $\mathrm{T}$ cell activation and promote TCR-dependent $\mathrm{CD}^{+} \mathrm{T}$ cell proliferation (14). Additionally, we recently demonstrated that cKO in DCs promotes Th1 differentiation and IFN- $\gamma$ production via upregulation of costimulatory molecules in PTPN2-deficient DCs (24).

Here, we demonstrate again that PTPN2 deletion in T cells and DCs increased IFN- $\gamma$ production, which promoted the differentiation and activation of $\mathrm{CD} 4^{+} \mathrm{Th} 1$ cells and increased the cytotoxic ability of $\mathrm{CD}^{+} \mathrm{T}$ cells. Of key importance to the therapeutic potential to inhibit PTPN2 function, however, is the fact that the highly increased frequency of granzyme B-producing, PTPN2-deficient $\mathrm{CD}^{+} \mathrm{T}$ cells was mainly confined to the tumor tissues, whereas levels in healthy tissues from tumor-bearing mice were only slightly increased compared with levels in WT controls. This suggests that specific antigen stimulation is necessary for the boosted $\mathrm{CD} 8^{+} \mathrm{T}$ cell response and that $\mathrm{T}$ cell-specific PTPN2 depletion or inhibition would probably not affect nontumor tissues. This unveils a therapeutic rationale for the use of T cell-specific PTPN2 inhibitors as an adjuvant therapy in CRC.

In agreement with the tumor-specific $\mathrm{CD} 8^{+} \mathrm{T}$ cell activation in the absence of PTPN2 in T cells or DCs, we observed highly increased intratumoral infiltration of PTPN2-deficient CD8 ${ }^{+} \mathrm{T}$ cells. IFN- $\gamma$-inducible chemokines, specifically CXCL9, CXCL10, and CXCL11 and their receptor CXCR3, have been described as important modulators of $\mathrm{CD}^{+} \mathrm{T}$ cell recruitment to tumor tissue (27-32). Here, we found that PTPN2 deletion in T cells and enhanced IFN- $\gamma$ signaling resulted in increased mRNA expression of many chemokines, but most significantly IFN- $\gamma$-inducible CXCL9 and CXCL11 and their receptor CXCR3, within the tumor tissue, highlighting the enhanced potential to attract and accumulate cytotoxic T cells within the tumor tissue. Hence, PTPN2 deficiency in T cells not only enhances activity and cytotoxicity of tumor-infiltrating $\mathrm{T}$ cells, but also facilitates their chemotaxis to the tumor.

PTPN2 deletion caused $C D 8^{+} \mathrm{T}$ cells to be readily activated and enhanced their cytotoxicity and infiltration into tumor sites. In concurrence with the inverse correlation between PTPN2 and PD-1 in human CRC, we demonstrated that absence of PTPN2 in T cells promoted the efficacy of anti-PD-1 therapy in murine CRC, resulting in complete tumor clearance. In line with an increased and sustained anti-PD-1 response, gene expression profiling revealed increased mRNA expression of $\mathrm{Cxcr} 3$ and $\mathrm{Cxcr5}$ - chemokine receptors crucial for efficient anti-PD-1 responses $(30,33)$ and memory formation $(34,35)$, respectively. Of importance for potential therapeutic strategies targeting PTPN2 in T cells is the memory formation that mediated the complete clearance of secondary tumors in mice lacking PTPN2 in T cells, indicating that PTPN2 deletion or inhibition might not only serve as a potential treatment in primary CRC, but might also significantly extend relapse-free survival.

Our data indicate that targeting PTPN2 represents a highly promising approach to enhance $\mathrm{T}$ cell tumor infiltration and tumor-specific cytotoxic activity and improve treatment response in tumors that are refractory to checkpoint inhibitors because of low immunogenicity. Additionally, our data suggest that targeting PTPN2 may prolong tumor-free survival via development of immune memory formation. 
A

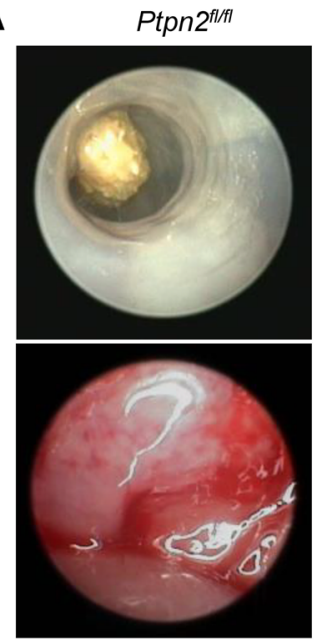

Ptpn2 $2^{\text {fl/fil }} \mathrm{Cd} 11 \mathrm{C}^{\mathrm{Cre}+/}$

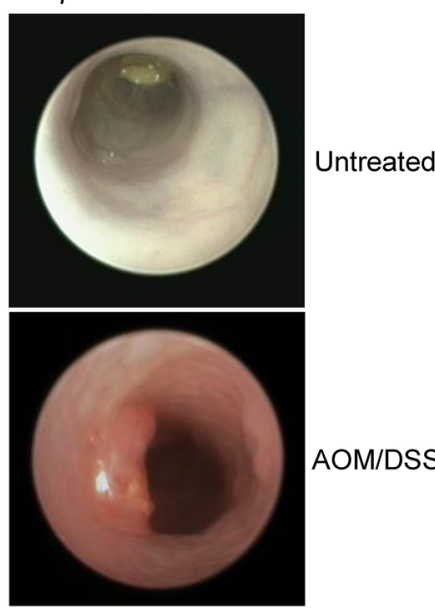

D

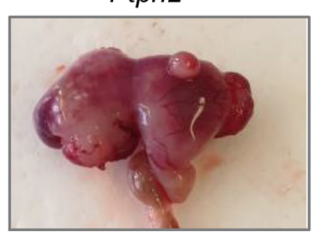

Ptpn2 ${ }^{\mathrm{fHfl}} \mathrm{Cd} 11 \mathrm{C}^{\mathrm{Cre}+/}$

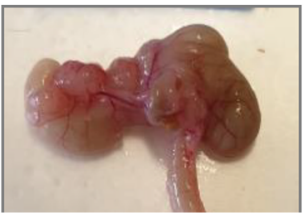

$\mathbf{F}$

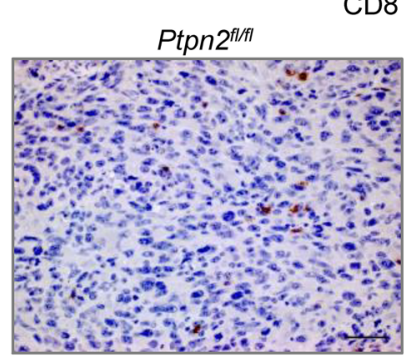

G

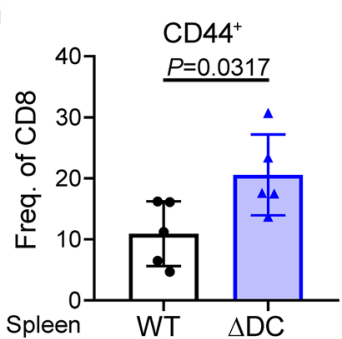

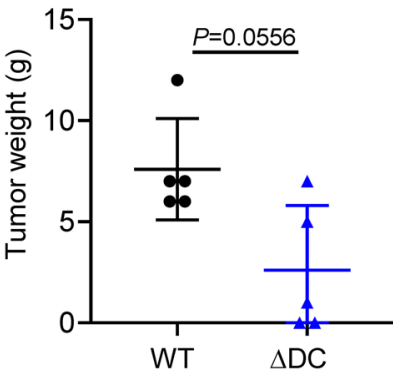

CD8 staining

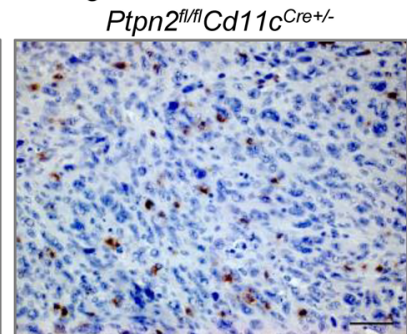

H

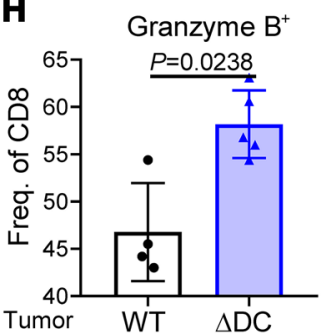

B

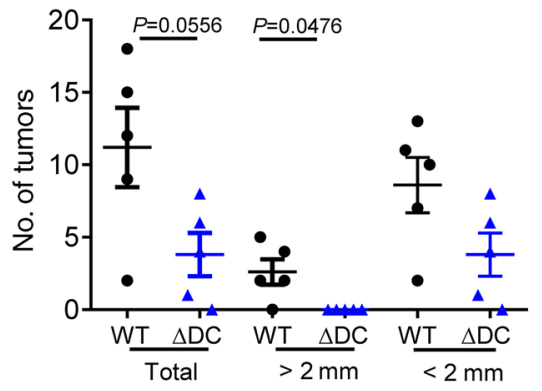

C

$\mathrm{CD}^{+}$

$\mathrm{CD} 44^{+}$
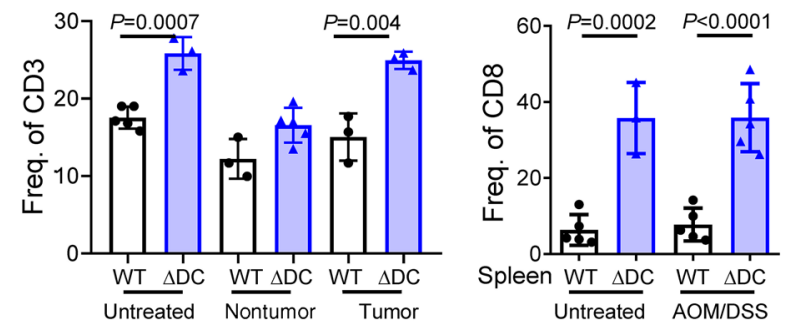

$\mathbf{E}$

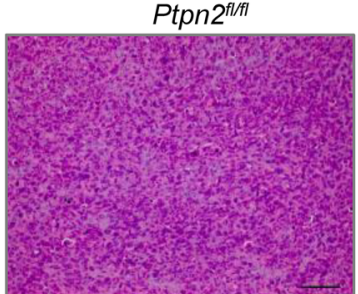

Ptpn2 ${ }^{\mathrm{fHIf}} \mathrm{Cd} 11 \mathrm{c}^{\mathrm{Cre}+/-}$

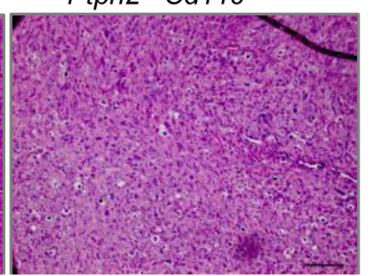

$\mathrm{CD}^{+}$
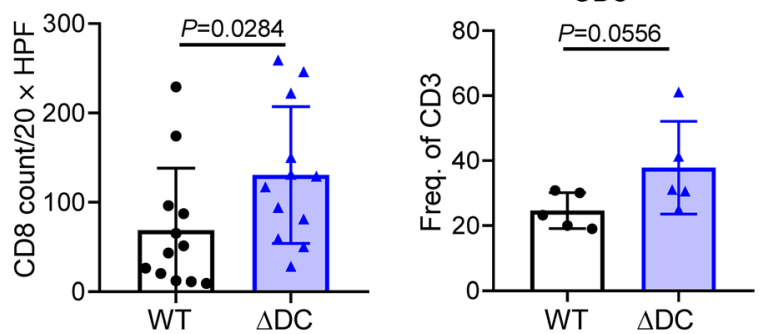

$\mathrm{IFN} \gamma^{+}$

$\mathrm{TNF}^{+}$

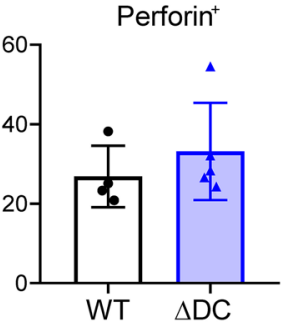

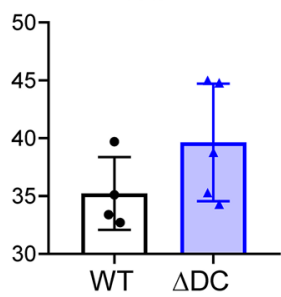

Figure 7. PTPN2 deletion in DCs leads to reduced tumor load in the AOM/DSS and orthotopic injection models. Tumor development was induced in WT mice ( $n=5$ mice) and their Ptpn2 $2^{f l / f l} C d 11 c^{\text {Cre+l- }}$ littermates $(\triangle \mathrm{DC})(n=5$ mice) using the AOM/DSS model and the cecal injection model. Mixed WT controls (Ptpn2 ${ }^{f / / f l} \mathrm{Cd} 4^{\text {Cre- }}$ and Ptpn2 $2^{f l / f l} C d 11 c^{C r e-}$ ) were used in these experiments, thus, WT quantification in $\mathbf{F}$ is the same as the WT quantification in Figure 4C.

(A) Representative colonoscopy images. (B) Quantification of the total tumor numbers and according to tumor size. $P$ values were determined by 2-tailed Mann-Whitney $U$ test. Flow cytometry analysis was performed on healthy colon, inflamed nontumor, and inflamed tumor tissue. (C) Frequencies of CD8 ${ }^{+}$ T cells (colon) and effector/memory CD8 ${ }^{+}$CD $44^{+} T$ cells (spleen). $P$ values were determined by 2 -tailed Mann-Whitney $U$ test. (D) Gross images of ceca

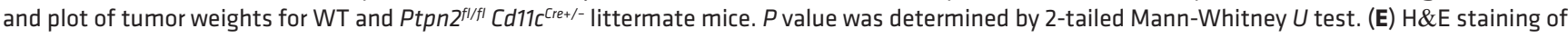
tumor tissue from WT and Ptpn $2^{f / f l} C d 11 c^{C r e+/-}$ mice. Scale bars: $100 \mu \mathrm{m}$. (F) Representative images and analysis of IHC and flow cytometric frequency of CD8 staining in WT and Ptpn $2^{f / f l} C d 11 c^{C r e+/-}$ mouse tumor tissue. Scale bars: $50 \mu \mathrm{m}$. $P$ values were determined by 2-tailed Mann-Whitney $U$ test. (G and H) Frequencies of effector/memory CD8+CD44+ T cells (spleen) and the cytotoxicity markers granzyme B, perforin, IFN- $\gamma$, and TNF (tumor). $P$ values were determined by 2-tailed Mann-Whitney $U$ test. Data represent the mean \pm SD. 
A
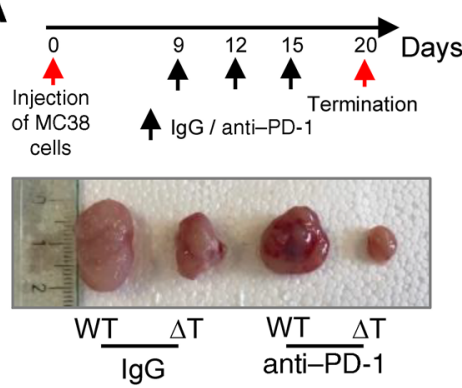

C

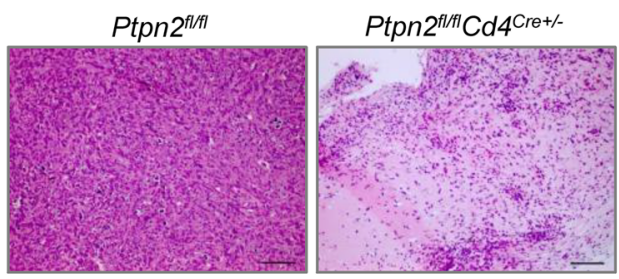

anti-PD-1 group

E

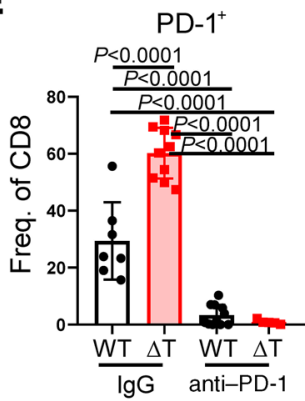

B

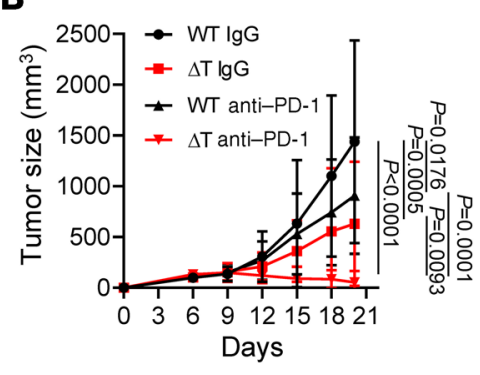

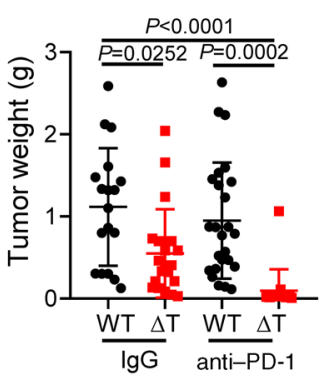

D

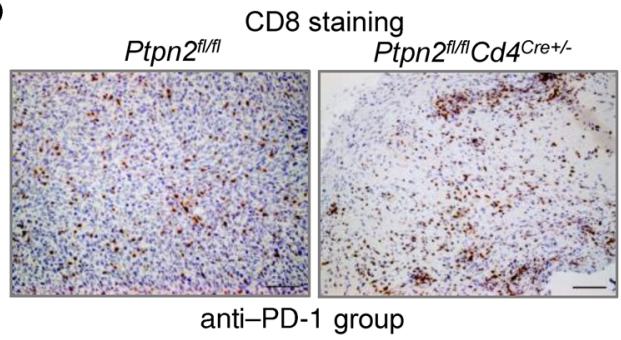

$\mathrm{CD}^{+}$

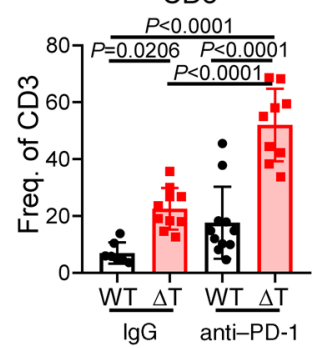

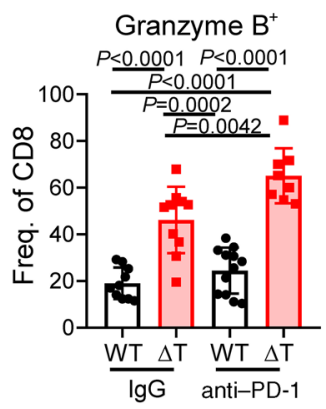

Perforin $^{+}$

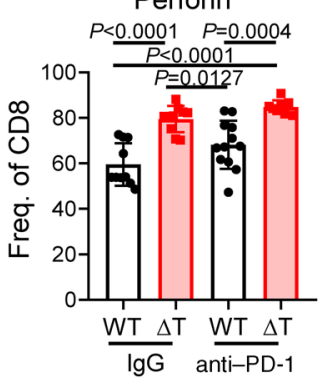

$\mathbf{F}$
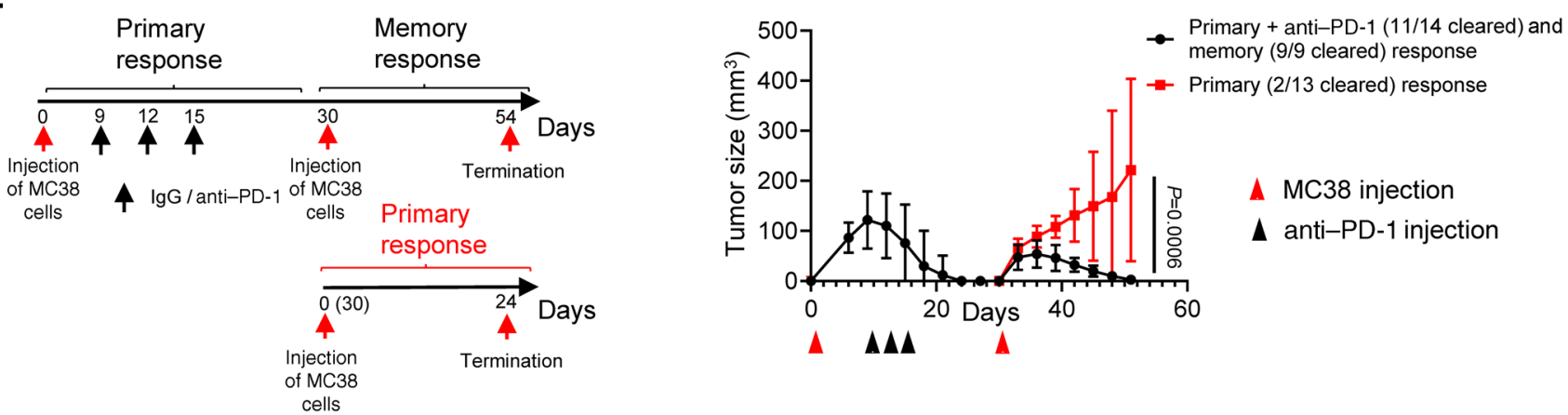

Figure 8. PTPN2 deficiency and anti-PD-1 therapy lead to an enhanced synergetic antitumor response. (A) Schematic overview of the treatment and representative gross images of tumors from WT and Ptpn2 $2^{f / f l} \mathrm{Cd} 4^{\text {Cre+t- }}$ mice treated with IgC control or anti-PD-1 antibody. (B) Tumor development and weights ( $n=10$ mice and 20 tumors; $n=2$ independent experiments). $P$ values were determined by 1-way ANOVA with Tukey's multiple-comparison test. (C) H\&E staining of tumor tissue from WT and Ptpn $2^{f^{f / f f}} \mathrm{Cd} 4^{\mathrm{Cre+}+-}$ mice. Scale bars: $100 \mu \mathrm{m}$. (D) Representative images of IHC staining and flow cytometric frequency of CD8 staining of WT and Ptpn $2^{f / f l} \mathrm{Cd}^{\mathrm{Cre+} /-}$ mice tumor tissue. Scale bars: $100 \mu \mathrm{m}$. 1-way ANOVA with Tukey's multiple-comparison test. (E) Frequencies of PD-1, CD44, granzyme B, and perforin on $C D 8^{+} T$ cells from IgG control and anti-PD-1 groups. $P$ values were determined by 1-way ANOVA with Tukey's multiple-comparison test. (F) Outline of the primary and memory response experiment and tumor development curve for Ptpn $2^{f l / f l} \mathrm{Cd} 4^{\mathrm{Cr} \mathrm{ret}^{-}-}$mice (first primary response, $n=7$ mice and 14 tumors; memory $n=7$ mice and 9 tumors; second primary response $n=13$ mice and 13 tumors; $n=2$ independent experiments). $P$ values were determined by 1-way ANOVA with Tukey's multiple-comparison test. Data represent the mean \pm SD.

\section{Methods}

Human samples. Paraffin-embedded human tissue slides used for PTPN2 and p-STAT1 were obtained from the Translational Research Center in Erlangen, Germany. RNA analysis was performed by the Translational Research Center in Erlangen, Germany, as described previously (36). A list of primers and probes can be found in Supplemental Table 1. Cryopreserved nontumor and colon tumor tissues for the phosphatase activity assay and paraffin-embedded primary colon tumor, liver, and lung metastasis tissue slides for PTPN2, PD-1, and CTLA4 IHC stainings were obtained from the Pathology Department of the University Hospital Zurich (Zurich, Switzerland). The tissue microarray slides containing melanoma tissue for PTPN2 and PD1 staining were obtained from the Department of Dermatology of the University Hospital Zurich. 
Phosphatase activity assay. Samples for phosphatase activity measurements were homogenized using the genteMACS tissue homogenizer (Miltenyi Biotec), and proteins were per-cleared using sepharose $\mathrm{G}$ beads (GE Healthcare) and then incubated overnight $(\mathrm{o} / \mathrm{n})$ at $4^{\circ} \mathrm{C}$ on a rocker with $10 \mu \mathrm{g} / \mathrm{mL}$ rabbit anti-PTPN2 (Calbiochem; clone CF4-1D). Beads were added after o/n incubation and left on the rocker for 1 hour at $4^{\circ} \mathrm{C}$. Precipitates were washed with PBS and phosphatase activity measured using the EnzChek Kit (Thermo Fisher Scientific). Fluorescence units (FU) were measured every 10 minutes on a Synergy HT plate reader using Gen5 software (both from BioTek Instruments).

Mice. C57BL/6N mice carrying Cre under the $C d 4$ promoter were purchased from Taconic, CD11cCre-GFP line 4097 mice from The Jackson Laboratory, Ptpn $2^{f / f l}$ mice from EUCOMM (Germany), APCmin mice from The Jackson Laboratory, Rag2 ${ }^{-/}$mice from Charles River Laboratories, and OT1/J mice from The Jackson Laboratory. All mice were kept in specific pathogen-free conditions. Ptpn $2^{f / f l}$ mice were bred with $C d 4^{\text {Cre }}$ mice to obtain specific PTPN2 deletion in T cells (17) and with $C d 11 c^{C r e-G F P}$ mice to create specific deletion of PTPN2 in DCs. Mice heterozygous for $C d 4^{\mathrm{Cre}}\left(\mathrm{Ptpn} 2^{\mathrm{fl} / \mathrm{fl}} \mathrm{Cd} 4^{\mathrm{Cret} / \mathrm{-}}\right)$ or $\mathrm{Cd} 11 \mathrm{C}^{\mathrm{Cre}}$ $\left(P t p n 2^{f / f l} C d 11^{C r e+/}\right)$ were bred with $P t p n 2^{f / / f l}$ (WT) mice to obtain $\mathrm{Cr}^{+/-}$

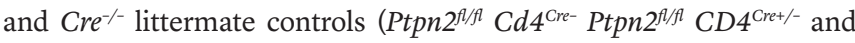
Ptpn $2^{f / / f l} \mathrm{Cd} 11 \mathrm{Cr} \mathrm{C}^{-} \mathrm{Ptpn} 2^{f / / f l} \mathrm{Cd} 11 c^{\mathrm{Cret} /-}$, respectively). Males and females between 8-12 weeks of age were used for all experiments, except for $\mathrm{AOM} / \mathrm{DSS}$ tumor induction, for which only females were used. Littermate controls were used for all experiments. Mixed Ptpn $2^{f / f l} C d 4^{\mathrm{Cre}-}$ and $P t p n 2^{f / f l} C d 11 c^{C r e-}$ control mice (referred to as Ptpn $2^{f / / f l}$ or WT controls) were used for AOM/DSS and cecal injection tumor models to minimize the number of animals required. Whenever mixed control animals were used, both $\mathrm{Ptpn} 2^{\mathrm{fl} / \mathrm{fl}} \mathrm{Cd} 4^{\mathrm{Cret}+-}$ and $\mathrm{Ptpn} 2^{\mathrm{fl} / \mathrm{fl}} \mathrm{Cd} 11^{\mathrm{Cret} /-}$ groups were included in the same experiment and run in parallel. Experiments with mixed control groups are indicated in the figure legends.

Tumor models and treatments. Colitis-associated tumors were induced as described previously (19) by administering DSS (MP Biomedicals) in the drinking water and injecting AOM (MilliporeSigma; $10 \mathrm{mg} / \mathrm{kg}$ in saline). Control mice received water and were injected with saline. Mice were euthanized 10 days after the last DSS treatment. After the third DSS cycle and at the end of the experiment, mice were anesthetized by i.p. injection of ketamine (90-120 mg/ $\mathrm{kg}$ body weight; Vetoquinol) and xylazine ( $8 \mathrm{mg} / \mathrm{kg}$ body weight; Bayer), and a colonoscopy was performed to monitor tumor growth.

For injection models, MC38-GFP (donated by Lubor Borsig, Institute of Physiology, University of Zurich, Zurich, Switzerland) or B16-F10 tumor cells were suspended in culture medium, mixed 1:1 with Matrigel and 300,000 cells, and injected into the cecal wall or s.c. into the mouse flank. Mice were euthanized 2-3 weeks after injection. In the s.c. model, tumor development was measured every 3 days using a digital caliper. Tumor volume was calculated using the ellipsoid formula: $4 / 3 \times 3.14 \times$ length $/ 2 \times(\text { width } / 2)^{2}$, where the shorter dimension was used as the width and depth. Mice were euthanized when the volume reached $1 \mathrm{~cm}^{3}$ or the length reached $2 \mathrm{~cm}$.

$\mathrm{CD}^{+} \mathrm{T}$ cells depletion was performed using anti-CD8 (Lyt 3.2) antibody (Bio X Cell; clone 53-5.8) or IgG isotype control (Bio X Cell; clone HRPN). Antibodies were injected i.p. in a concentration of 200 $\mathrm{mg} / \mathrm{mouse}$ on day -3 and $100 \mathrm{mg} /$ mouse on day 0 and then once weekly thereafter. $\mathrm{CD} 4^{+} \mathrm{T}$ cells depletion was performed using an anti-CD4 antibody (Bio X Cell; clone GK 1.5) or an IgG isotype control
(Bio X Cell; clone LTF-2). Antibodies were injected i.p. at a concentration of $200 \mathrm{mg} / \mathrm{mouse}$ on day -3 , day 0 , and every 3 days thereafter. PD-1 blockade was performed in the s.c. tumor model and achieved by i.p. injection of $200 \mathrm{mg} / \mathrm{mouse}$ anti-PD-1 (CD279) antibody (Bio X Cell; clone 29F.1A12) or IgG isotype control (Bio X Cell; clone 2a3) on days 9,12 , and 15 after injection of MC38 cells.

For adoptive transfer, 500,000 CD8 $8^{+}$T cells from Ptpn $2 \mathrm{fl} / \mathrm{fl}$ and

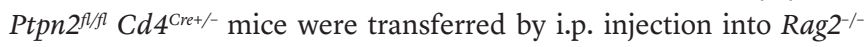
mice on day 6 after the s.c. MC38 tumor injection.

Histology. Tumor and tumor-adjacent tissue from patients, the most distal colon sections from the AOM/DSS experiment mice, and tumors from s.c. injection experiments were used for H\&E staining, IHC, and immunofluorescence (IF).

Tissues for IF were deparaffinized and antigens retrieved using citrate buffer, pH 6.0 (Dako), at $98^{\circ} \mathrm{C}$ for 30 minutes. Slides were maintained in PBS with 10\% goat serum, 10\% BSA, and 0.3\% Triton $\mathrm{X}$ (MilliporeSigma) for 1 hour at room temperature (RT) to block the nonspecific binding. Primary antibodies for IF were diluted in $1 \%$ BSA, $1 \%$ goat serum, and $0.3 \%$ Triton $\mathrm{X}$ and incubated overnight at $4^{\circ} \mathrm{C}$. A list of antibodies is provided in Supplemental Table 2. Slides were washed with $3 \%$ milk and PBS solution. Secondary goat antirabbit AF647 (Thermo Fisher Scientific) and goat anti-mouse AF594 (Thermo Fisher Scientific) were diluted in 1\% BSA, 1\% goat serum, and $0.3 \%$ Triton $\mathrm{X}$, and the slides were incubated for 1 hour, washed with PBS, and then mounted. Fluorescence-labeled tissue sections were examined using the Leica SP8 Upright microscope and Leica LAS $\mathrm{X}$ and LAS AF Lite software.

H\&E staining was performed according to standard procedures. Tissues for IHC were deparaffinized and antigens retrieved using citrate buffer, $\mathrm{pH} 6.0$ (Dako), at $98^{\circ} \mathrm{C}$ for 30 minutes. Inhibition of endogenous peroxidases was performed by incubation of tissue slides in $0.9 \%$ hydrogen peroxide for 15 minutes at RT. Horse serum (2.5\%) for blockage of nonspecific binding was used for p-STAT1 and CD4 staining for 1 hour and for PTPN2 in human tissue for 2 hours at RT. For CD3, CD8, PD-1, and CTLA4 staining, 3\% BSA blocking solution was used for 1 hour. Primary antibodies were diluted in blocking solutions and slides incubated overnight at $4^{\circ} \mathrm{C}$. A list of antibodies is provided in Supplemental Table 2. A secondary HRP-labeled antibody (Vector Laboratories, ImmPRESS) was applied for IHC samples for 1 hour at RT, and staining was visualized using the DAB ImmPACT Peroxidase Substrate Brown Kit (Vector Laboratories). IHC samples were counterstained with hematoxylin, dehydrated, and mounted. Tissue sections were examined using the Zeiss Axio Imager Z2 and Zeiss 2.6 (Blue Edition) software.

$q P C R$. Healthy and tumor tissues $(0.5 \mathrm{~cm})$ were homogenized using gentleMACS (Miltenyi Biotec), and RNA was isolated using the Maxwell RSC simplyRNA Tissue Kit (Promega) according to the manufacturer's instructions. RNA concentration was measured using absorbance at 260 and $280 \mathrm{~nm}$. cDNA was synthesized using the high-capacity cDNA Reverse Transcription Kit (Thermo Fisher Scientific) according to the manufacturer's instructions. qPCR was performed using FAST qPCR Master Mix and predesigned TaqMan assays (Thermo Fisher Scientific) on a QuantStudio 6 system using QuantStudio software (Thermo Fisher Scientific). Mouse GAPDH was used as an endogenous control. Relative expression levels were calculated according to the $\Delta \Delta \mathrm{Ct}$ method, and samples were measured in triplicate. 
Flow cytometric analysis. Spleen, mesenteric and skin draining LNs, colon lamina propria lymphocytes (LPLs), and tumor cells were used for flow cytometric analysis. Single-cell suspensions from spleen, LNs, and LPLs were prepared as described previously (37). Cecal and s.c. tumors were cut into pieces of approximately $0.5 \mathrm{~mm}^{3}$ in size and digested in $6 \mathrm{~mL}$ containing $0.5 \mathrm{mg} / \mathrm{mL}$ collagenase type IV (MilliporeSigma) and $0.05 \mathrm{mg} / \mathrm{mL}$ DNAse I (Roche) solution for 10 minutes on a shaker $(300 \mathrm{rpm})$ at $37^{\circ} \mathrm{C}$. Cells were homogenized by passage through an 18 gauge $\times 1.5$ inch syringe and centrifuged for 10 minutes at $4^{\circ} \mathrm{C}$ and $300 \mathrm{~g}$. Single-cell suspensions were stained and restimulated as described previously (38). The antibodies used are listed in Supplemental Table 3.

RNA isolation, sequencing, transcript quantification, and differential expression analysis. Healthy, inflamed nontumor, and tumor tissues from AOM/DSS-treated and water-treated control Ptpn $2^{f / f l}$ and $P t p n 2^{f / / f}$ $C d 4^{\text {Cret/- }}$ mice were used for RNA-Seq analysis. RNA isolation and sequencing were performed by Microsynth.

Transcripts were quantified with kallisto 0.44.0 (39) using the GRCm38 reference. Genes with a total transcript abundance quantification of less than 10 were discarded from the downstream analysis. Differential expression analysis was performed in R (40) with DESeq2 (41) using the following design formula: genotype $\times$ treatment + tissue:genotype. Shrinkage of $\log _{2}$ fold changes was estimated using apeglm (42). All sequencing data can be found in the ArrayExpress database (accession number E-MTAB-9629).

In vitro killing assay. For the in vitro killing assay 50,000 MC38GFP cells were stained with CellTrace Violet (Invitrogen, Thermo Fisher Scientific), mixed with 50,000 MC-38-OVA-GFP cells stained with CellTrace Yellow (Invitrogen, Thermo Fisher Scientific), and incubated for 24 hours at $37^{\circ} \mathrm{C}$. Cells were then kept alone or cocultured with enriched CD $8^{+} \mathrm{T}$ cells from spleens of either Ptpn $2^{f / f l} \mathrm{Cd} 4^{\mathrm{Cre}-}$ OT I or Ptpn $2^{f / f l} \mathrm{Cd} 4^{\mathrm{Cret} /-}$ OT I mice for 24 hours in a $37^{\circ} \mathrm{C}$ incubator. $\mathrm{CD}^{+} \mathrm{T}$ cells were enriched using the EasySep CD8 ${ }^{+} \mathrm{T}$ Cell Enrichment Kit (STEMCELL Technologies). Apoptosis was then detected using the Annexin V Kit - DY-634 Antibody (Abcam) and the Zombie NIR Fixable Viability Kit (BioLegend).

Statistics. For 2-group comparisons, a nonparametric, 2-tailed Mann-Whitney $U$ test was performed, and for comparison of 3 or more groups, a 1-way ANOVA followed by Tukey's multiple-comparison test was used. All experiments, which were performed in parallel, were analyzed together and later separated into different graphs to demonstrate results in different figures. For RNA-Seq analysis, a false sign rate was used to determine significance. A $P$ value of less than 0.05 was considered statistically significant.

Study approval. All tissues were collected in accordance with local ethics regulations, and patients provided written informed consent prior to sample collection. The study was approved by the Cantonal Ethics Committee of the Canton Zürich, Switzerland (approval no. 2018-02193 and KEK-ZH-647) and the local ethics committee of the Friedrich-Alexander-University (FAU) of Erlangen-Nürnberg. All animal experiments were performed according to Swiss animal welfare legislation and approved by the local veterinary office (Veterinäramt des Kantons Zürich; license nos. 239/2016 and 179/2019).

\section{Author contributions}

EK performed data acquisition, analysis, and interpretation and wrote the manuscript. LH, AMA, PB, M. Schwarzfischer, RM, JC, KA, S. Lang, and EN acquired and analyzed data. VSS, M. Stürzl, AR, MT, AW, S. Leibl, and ML acquired patient samples, and interpreted data. GEL performed data analysis and interpretation. OB and GR interpreted data. M. Scharl conceived, designed, and supervised the study, interpreted data, and acquired funding. MRS designed and supervised the study and interpreted data.

\section{Acknowledgments}

Flow cytometry was performed in the flow cytometry facility at the University of Zurich. We thank Miro E. Raeber (Department of Immunology, University Hospital Zurich, Zurich, Switzerland) for his technical support with $\mathrm{CD} 4^{+} \mathrm{T}$ cell depletion experiments. This work was supported by grants from the Stiftung Experimentelle Biomedizin (to M. Scharl); the Swiss National Science Foundation (SNSF) (SNSF 314730_166381 and SNSF 320030_184753, to M. Scharl); the Novartis Foundation for Biomedical Research (to M. Scharl); and the Deutsche Forschungsgemeinschaft (DFG) (DFGFOR2438, to EN and M. Stürzl, and DFG-TRR241, to M. Stürzl).

Address correspondence to: Michael Scharl, Department of Gastroenterology and Hepatology, University Hospital Zurich, Rämistrasse 100, 8091 Zurich, Switzerland. Phone: 41.44.255.3419. Email: Michael.Scharl@usz.ch.
1. Ciardiello D, et al. Immunotherapy of colorectal cancer: Challenges for therapeutic efficacy. Cancer Treat Rev. 2019;76:22-32.

2. Ganesh $\mathrm{K}$, et al. Immunotherapy in colorectal cancer: rationale, challenges and potential. Nat Rev Gastroenterol Hepatol. 2019;16(6):361-375.

3. Bray F, Ferlay J, Soerjomataram I, Siegel RL, Torre LA, Jemal A. Global cancer statistics 2018: GLOBOCAN estimates of incidence and mortality worldwide for 36 cancers in 185 countries. $C A$ Cancer JClin. 2018;68(6):394-424.

4. Montalban-Arques A, Scharl M. Intestinal microbiota and colorectal carcinoma: implications for pathogenesis, diagnosis, and therapy. EBioMedicine. 2019; carcinoma;48:648-655.

5. Rawla P, Barsouk A, Hadjinicolaou AV, Barsouk A. Immunotherapies and targeted therapies in the treatment of metastatic colorectal cancer.
Med Sci (Basel). 2019;7(8):E83.

6. Umezawa S, et al. Chemoprevention of colorectal cancer: Past, present, and future. Cancer Sci. 2019;110(10):3018-3026.

7. Passarelli A, Mannavola F, Stucci LS, Tucci M, Silvestris F. Immune system and melanoma biology: a balance between immunosurveillance and immune escape. Oncotarget. 2017;8(62):106132-106142.

8. Givechian KB, Garner C, Benz S, Song B, Rabizadeh S, Soon-Shiong P. An immunogenic NSCLC microenvironment is associated with favorable survival in lung adenocarcinoma. Oncotarget. 2019;10(19):1840-1849.

9. Kalyan A, Kircher S, Shah H, Mulcahy M, Benson A. Updates on immunotherapy for colorectal cancer. J Gastrointest Oncol. 2018;9(1):160-169.

10. Arora SP, Mahalingam D. Immunotherapy in colorectal cancer: for the select few or all? J Gas- trointest Oncol. 2018;9(1):170-179.

11. Manguso RT, et al. In vivo CRISPR screening identifies Ptpn2 as a cancer immunotherapy target. Nature. 2017;547(7664):413-418.

12. LaFleur MW, et al. PTPN2 regulates the generation of exhausted $\mathrm{CD}^{+} \mathrm{T}$ cell subpopulations and restrains tumor immunity. Nat Immunol. 2019;20(10):1335-1347.

13. Wiede F, et al. PTPN2 phosphatase deletion in $\mathrm{T}$ cells promotes anti-tumour immunity and CAR T-cell efficacy in solid tumours. EMBO J. 3637;2019(2):e103637.

14. Wiede $\mathrm{F}$, et al. $\mathrm{T}$ cell protein tyrosine phosphatase attenuates $\mathrm{T}$ cell signaling to maintain tolerance in mice. J Clin Invest. 2011;121(12):4758-4774.

15. Scharl M, Hruz P, McCole DF. Protein tyrosine phosphatase non-receptor type 2 regulates IFN- $\gamma$ induced cytokine signaling in THP-1 monocytes. 
Inflamm Bowel Dis. 2010;16(12):2055-2064.

16. Scharl M, et al. Protein tyrosine phosphatase N2 regulates $\mathrm{TNF} \alpha$-induced signalling and cytokine secretion in human intestinal epithelial cells. Gut. 2011;60(2):189-197.

17. Spalinger MR, et al. PTPN2 controls differentiation of CD4(+) T cells and limits intestinal inflammation and intestinal dysbiosis. Mucosal Immunol. 2015;8(4):918-929.

18. Heinonen KM, et al. T-cell protein tyrosine phosphatase deletion results in progressive systemic inflammatory disease. Blood. 2004;103(9):3457-3464.

19. Spalinger MR, et al. PTPN2 regulates inflammasome activation and controls onset of intestinal inflammation and colon cancer. Cell Rep. 2018;22(7):1835-1848.

20. Alcantara $\mathrm{M}$, et al. Clinical and biological features of PTPN2-deleted adult and pediatric T-cell acute lymphoblastic leukemia. Blood $A d v$. 2019;3(13):1981-1988.

21. Wiede F, et al. PTPN2 regulates $\mathrm{T}$ cell lineage commitment and $\alpha \beta$ versus $\gamma \delta$ specification. J Exp Med. 2017;214(9):2733-2758.

22. Sharma S, Zhu J. Immunologic applications of conditional gene modification technology in the mouse. Curr Protoc Immunol. 2014;105:10.34.1-10.34.13.

23. Lee PP, et al. A critical role for Dnmt1 and DNA methylation in T cell development, function, and survival. Immunity. 2001;15(5):763-774.

24. Hering L, et al. Protein tyrosine phosphatase non-receptor type 2 function in dendritic cells is crucial to maintain tissue tolerance. Front Immunol.2020;11:1856.
25. de Vries NL, Swets M, Vahrmeijer AL, Hokland $\mathrm{M}$, Kuppen PJ. The immunogenicity of colorectal cancer in relation to tumor development and treatment. Int J Mol Sci. 2016;17(7):E1030.

26. Van der Jeught K, Xu HC, Li YJ, Lu XB, Ji G. Drug resistance and new therapies in colorectal cancer. World J Gastroenterol. 2018;24(34):3834-3848.

27. Kistner L, Doll D, Holtorf A, Nitsche U, Janssen KP. Interferon-inducible CXC-chemokines are crucial immune modulators and survival predictors in colorectal cancer. Oncotarget. 2017;8(52):89998-90012.

28. Metzemaekers M, Vanheule V, Janssens R, Struyf $\mathrm{S}$, Proost p. overview of the mechanisms that may contribute to the non-redundant activities of interferon-inducible CXC chemokine receptor 3 ligands. Front Immunol. 2017;8:1970.

29. Tokunaga R, et al. CXCL9, CXCL10, CXCL11/ CXCR3 axis for immune activation - a target for novel cancer therapy. Cancer Treat Rev. 2018;63:40-47.

30. Chow MT, et al. Intratumoral activity of the CXCR3 chemokine system is required for the efficacy of antiPD-1 therapy. Immunity. 2019;50(6):1498-1512.e5.

31. Peng D, et al. Epigenetic silencing of TH1-type chemokines shapes tumour immunity and immunotherapy. Nature. 2015;527(7577):249-253.

32. Zingg D, et al. The histone methyltransferase Ezh2 controls mechanisms of adaptive resistance to tumor immunotherapy. Cell Rep. 2017;20(4):854-867.

33. Han $X$, et al. Role of CXCR3 signaling in response to anti-PD-1 therapy. EBioMedicine. 2019;48:169-177.
34. Im SJ, et al. Defining CD8 ${ }^{+} \mathrm{T}$ cells that provide the proliferative burst after PD-1 therapy. Nature. 2016;537(7620):417-421.

35. Perdomo-Celis F, Taborda NA, Rugeles MT. Follicular $\mathrm{CD}^{+} \mathrm{T}$ cells: origin, function and importance during HIV infection. Front Immunol. 2017;8:1241.

36. Naschberger E, et al. Matricellular protein SPARCL1 regulates tumor microenvironment-dependent endothelial cell heterogeneity in colorectal carcinoma. J Clin Invest. 2016;126(11):4187-4204.

37. Spalinger MR, et al. NLRP3 tyrosine phosphorylation is controlled by protein tyrosine phosphatase PTPN22. JClin Invest. 2016;126(5):1783-1800.

38. Spalinger MR, et al. Loss of PTPN22 abrogates the beneficial effect of cohousing-mediated fecal microbiota transfer in murine colitis. Mucosal Immunol. 2019;12(6):1336-1347.

39. Bray NL, Pimentel H, Melsted P, Pachter L. Near-optimal probabilistic RNA-seq quantification. Nat Biotechnol. 2016;34(5):525-527.

40. R Core Team. R: A language and environment for statistical computing. R Foundation for Statistical Computing, Vienna, Austria. 2019. https://www.R-project.org/. Accessed October 8, 2020.

41. Love MI, Huber W, Anders S. Moderated estimation of fold change and dispersion for RNA-seq data with DESeq2. Genome Biol. 2014;15(12):550.

42. Zhu A, Ibrahim JG, Love MI. Heavy-tailed prior distributions for sequence count data(12): removing the noise and preserving large differences. Bioinform. 2019;35(12):2084-2092. 